\title{
Mobile and Intelligent Sensing for Video Watermarking Using Spectral Centroid and Haar Wavelet Transformation on High Performace Computing
}

Venkatesh S ( $\sim$ venkyjep2019@gmail.com )

Jeppiaar Engineering College

Saravanakumar $\mathbf{R}$

Dayananda Sagar College of Engineering

SureshKumar M

Sri Sairam College of Engineering

sivakumar $B$

SRMIST: SRM Institute of Science and Technology

veeramakali T

Veltech: Vel Tech Rangarajan Dr Sagunthala R\&amp;D Institute of Science and Technology

\section{Research Article}

Keywords: Video Watermarking, Spectral Centroid, Haar Wavelet Transformation, Fireflies algorithm, SNR, HWT-DFT, OOMP.

Posted Date: April 16th, 2021

DOl: https://doi.org/10.21203/rs.3.rs-395999/v1

License: (c) (i) This work is licensed under a Creative Commons Attribution 4.0 International License.

Read Full License 


\section{Mobile and Intelligent Sensing for Video Watermarking Using Spectral Centroid and Haar Wavelet Transformation on High Performace Computing}

1.Dr. S. Venkatesh, Assistant Professor, Department of Computer Science and Engineering, Jeppiaar Engineering College, Chennai, India,E-Mail: venkyjep2019@gmail.com

2. Dr.R.Saravanakumar,Professor, Department of CSE,Dayananda sagar Academy of Technology and Management, Kanakkapura road, Bangalore- 560082

dr.saravanakumar@dsatm.edu.in

3. M.SureshKumar,Associate Professor,Department of Information Technology

Sri SaiRam Engineering College,Chennai.

Sureshkumar.IT@sairam.edu.in

4.B.Sivakumar,Associate Professor, Department of Computer Science and Engineering SRMIST-KTR,Chennai-India,sivakumb2@srmist.edu.in

5. T.Veeramakali ,Associate Professor, Department of Computer Science and Engineering, School of Computing,Vel Tech Rangarajan Dr.Sagunthala R\&D Institute of science and Technology,Avadi, Chennai,drveeramakalit@veltech.edu.in

\footnotetext{
Abstract

Some technologies are technologically advanced to provide security from illegal copying. Two complementary methods are encryption and watermarking. Encryption safeguards the information throughout the communication from the sender to the receiver. The data might present a distorted image after receipt and subsequent decryption. Watermarking complements encryption through embedding data openly into the image. Therefore, the watermark continuously remains existing in the data. A digital watermark is a category of indication secretly entrenched in a noise-tolerant signal similar to audio or else image information. It is indeed applied to distinguish copyright possession of such signal. Computeraided hiding of the given digitized information in a carrier is known as watermarking.

Digital watermarks possibly will be employed to validate the authenticity or integrity of a carrier signal or to determine source uniqueness. It is evidently applied for determining copyright contraventions and aimed at banknote verification. Analogous to traditional watermarks, digital watermarks are unique only
} 
beneath certain conditions. Once a digital watermark varies a carrier in a manner that it turns out to be noticeable, formerly it is of no use. The media will be visible by traditional watermarks (similar to images or else video) but the signal might be pictures, video, audio, texts or 3D models in digital watermarking. A signal can transmit some different watermarks at the equivalent time.

Image watermarking is achieved in this study using two methods known as Hidden Markov TreeContourlet Wavelet Transform (HMT-CWT) and Haar wavelet transform - Discrete Fourier transform (HWT-DFT). In the next HWT-DFT method, a video is given as an input and it is split into two halves (audio and image). The audio is de-watermarked through Spectral Centroid Wavelet Transform and enhanced by utilizing Firefly procedure. The images is handled through HWT in addition to DFT. Then the output watermarked images and audio combined together to form a watermarked video. The obtained video is de-watermarked to produce the original copy of the video. The process of getting back the original copy by removing the watermark from the video is called as de-watermarking.

\section{Keywords:}

Video Watermarking, Spectral Centroid, Haar Wavelet Transformation, Fireflies algorithm, SNR, HWT-DFT, OOMP.

\section{Introduction}

The previous a long time have seen an outstanding progression in virtual communication and computer era. The blessings they provide are an green transmission of facts, high computing power, availability of excessive bandwidth, clean enhancing of virtual contents, etc.

It is now clean to copy virtual content with none loss inside the great of the virtual media. The boom in this era has additionally conveyed unique issues besides its benefits. The hassle of ease to copy virtual content material, edit, and transfer, it is able to violate copyright protection. Digital watermarking has been advised as an advanced approach to affirm possession of virtual data (Katariya 2012).

Watermark evidence is entrenched into the original information (crowd indication) in such a way that it stays existing on circumstance that the clear exceptional of the content of host sign is at an appropriate level. The original records owner demonstrates his/her possession by using extracting the watermark statistics from the watermarked content (watermarked signal) in case of multiple ownership claims.

The strategies used for information protection are cryptography, steganography and watermarking. Cryptography offers with securing contents of a message (host signal), after encryption, the message seems like noise and is useless. However, encryption structures do not completely solve the security hassle; due to the fact once decryption is performed. 
There is not any control over the dissemination of the facts (Barni and Bartolini 2004 and Lu 2004). Hence, a method is required which continually offer safety to host signal. Steganography and watermarking are seemed as the techniques which give protection to host sign all the time. The basic idea of steganography is to embed a secret message in a media that is interpreted through the supposed user only.

Steganography pacts with hiding a message, however watermarking have a further obligation of securely hiding the message, in such a way that the message cannot interfere. A right Steganographic scheme can entrench a massive amount of data without a perceptual degradation to multimedia statistics.

On the alternative hand, a great watermarking scheme would entrench records that couldn't be changed or eliminated without growing the multimedia signal unusable. A watermarking gadget involves a tradeoff among imperceptibility, robustness and embedding capacity (Barni and Bartolini 2004). It includes steps, viz. Watermark embedding and watermark detection/extraction. An encryption key is a mystery that is used in cryptography to make hidden information more secure.

The watermark records may be a random number that is generated the use of a mystery key which is known as the seed of watermark. Embedding is a process with the aid of which watermark records is fused with the original signal (additionally referred to as host signal) to offer a watermarked signal. Detection manner tests the presence of watermark data within the watermarked sign, however the extraction system unearths the watermark records from the watermarked signal. User keys are mystery which is used for watermark embedding. These keys can be watermark length, watermark location, watermark scaling factor, etc.

Digital Watermarking is the phenomena of hiding/embedding digital statistics right into a host sign in such a way that sign keeps its perceptual transparency (Bender et al 1996). The resulting sign is called a watermarked signal. The embedded records may be a chain of random numbers, text or an photograph and is called a watermark. The embedded data need to be recoverable via the authorized receiver even after the application of sign processing attacks on the watermarked signal. On the nature of host sign basis, watermarking is classified as picture, audio, and video watermarking. There are unique worries to these different codecs of statistics in watermarking system design.

In this chapter, audio and image watermarking using the Haar Wavelet Transform-Discrete Fourier Transform (HWT-DFT) are discussed.

\section{Literature Review}


Ritu Rawat et al (2016) reviewed the various watermarking technique and produced a brief introduction to these method employed for watermarking. Digital image watermarking became the vital research work for researches. This paper survived the spatial domain and frequency domain techniques of digital image watermarking. All techniques employed for watermarking had both merits and demerits. Still, digital image watermarking is a challenging work area for researchers in the field watermarking.

Parekh et al (2018) proposed an innovative work on watermarking by spatial domain approach using the matrix of the correlation coefficient. The suggested method could be categorized as blind watermarking (invisible). The performance of the recommended watermark was verified with simple attacks like compression and noise addition. The offered spatial domain watermarking could be employed for authentication of data.

Abraham et al (2016) proposed a paper on set of rules for embedding a photograph or logo in a colored photo. They endorsed two mask to provide less distracting to eyes of the human as embedding and repayment mask. In this approach, the information of the watermark was widely spread to enhance its stability against the attacks. They highlighted that the occurrence of $\mathrm{S}$ and $\mathrm{P}$ noise attack when the pixel was enhanced due to its nearby pixel which would be resolved by their methodology.

The implementation of mask aided them to have an even distribution of the modification that was carried out. The suggested algorithm was verified on many images. The imperceptibility and robustness of the Watermarked pictures have been experimentally assessed. The suggested methods delivered great excellence of images watermarked that could endure the right amount of attacks.

Su et al (2013) performed an analysis theoretically and proposed a new algorithm on color image blind watermarking. The suggested algorithm has greater watermark robustness and invisibility under the principle of intensification of embedded watermark capacity after performing the verification. The prominent aspects of their algorithm were: they embedded the watermark into the spatial domain on Discrete Cosine component instead of DCT domain, and their algorithm was highly efficient and simpler and could achieve the persistence of blind extracting watermark in the spatial domain.

Su et al (2017) proposed a blind watermarking established on DC coefficients in the spatial domain. The robustness and the invisibility of watermarks are increased by four times repeatedly inserting in the spatial domain with the principle of modifying DC coefficient in DCT domain. Furthermore, their method extracted the sub watermark by employing the extraction rules in the absence of the original watermark or host images. 
The optimum sub-watermarks are selected from the inserted watermark and then it combined with the final watermark to conglomerate the sub-watermarks. The suggested algorithm has robustness beside geometric attacks and standard image processing was investigated from the outcomes.

Pardhuet al (2016) concentrated on DWT and DCT frequency domain techniques and they successfully embedded and extracted the watermark into the image of the cover and showed no perceptual variation among the watermarked image and cover image, which fulfilled one of the chief necessities of the digital watermarking, i.e., imperceptibility.

In the application, diverse watermarked and cover images were considered. They calculated the SNR values for each image and stated that when the SNR increases the DATA for the images also increased. In their research, watermark and cover images were of the same size and if the watermark size was fewer than the image of the cover, then improved outcomes could be done to endure the watermarked image during image processing.

Susantoet al (2017) proposed watermarking technique with a blend of Haar Wavelet Transform (HWT)DCT on grayscale images with $512 \times 512$ pixels and had evaluated to investigate the performance. Every image showed a good CC and PSNR. The suggested algorithm was accomplished both of robustness and imperceptibility. Robustness verified by numerous spells of image processing, like Gaussian noise, unsharp filter, blurring, salt and pepper noise, JPEG Compression, cropping, low pass filtering, and mid filtering. The copyright extraction estimated by the coefficient of correlation, which was obtained with a mean of 0.8670 .

Kiran Kumar et al (2018) proposed added method for watermarking of the sound flag to mount a watermark plan in the vision of FFT strategies. Water stamp was injected into wave document ensuing to waning clamor, and illogical bearer was chosen somewhat than settle transporter. Their plan of injecting adjusted the watermark with the aim that the liveliness of the watermark was extended under the restriction of possession the sound-related relic as small as could be allowed.

They demonstrated that their watermarking plan was strong to normal flag preparing attacks and suggested strategy accomplished esteems of PSNR from $13 \mathrm{~dB}$ to $24 \mathrm{~dB}$ for several watermarked sounds. These results showed that their suggested strategy of watermarking could be a sensible competitor for copyright security of sound.

Sk et al (2018) developed the scheme for the protection of data in IoT applications. They stated that existing wireless technologies has no authentication of information and proprietorship despite providing data secrecy through encryption mechanisms. Thus the privacy and security of the data on the IoT 
applications were of core concern. This scheme was intended at providing ownership and data authentication by Play fair cipher variability.

The false positive singular value problem of breakdown based watermarking has received the resolution from the scheme. The suggested scheme was outclassing the contending methods concerning top signalto-noise proportion, minute fault rate correlation, and also evading false positives which shown from the experimental results.

Ahmad et al (2018) reported the feasibility of schemes in watermarking and perceptual image hashing on the bases of the print-scanned images. The comprehensive investigational analysis conducted and validated the capability of watermarking and perceptual image hashing on the bases of WAT to provide copyright and authentication security for print-scanned pictures, correspondingly. They projected a mixture hashing scheme with mess from ruler band 2 and ruler band three that has the competence to the acceptable rate of error less than $9 \%$ with segregation up to $15 \%$ of malicious manipulations. The investigational analysis of watermarking scheme bases has validated the great robustness of forensic watermarks in contradiction to the attack of print-scan.

Additionally, the improved versatile scheme exploited a reckless and computational meek algorithm which was appropriate for application in indicator held devices to offer instant image copyright and authentication protection for both print-scanned and digital-only images. They suggested that schemes to offer rotation on bases could be developed for robustness and enhance the balance among the discrimination and robustness strength of the hashing scheme while upholding the computational easiness and condensed hash size of the scheme.

Roopalakshmi (2018) proposed an approximation framework of position by content-based multimodal features, which safeguards the digital cinema counter to illicit camcorder captures in theaters. The investigational outcomes proved that the suggested framework could approximate the locations of the pirate. The proposed system can be employed for applications like sensor forensics, which efforts to recognize the procurement device that caught the movie unlawfully. She concluded with a scope to focus on refining the accuracy of estimation, seeing extra information about the geometry of theater like screen curvature, its aspect ratio and so on.

Ammar et al presented a complete framework for inaugurating the evidence of concept for saliency extraction earlier to entropic coding from the syntax elements of MPEG-4 AVC. The investigational validation carried out at two levels: applicative integration and ground-truth confrontation. The validation of ground truth was built on two criteria, Precision and Discriminance. 
Their outcome was bound by the reported experiments with some exemptions; approaches extracting saliency in the compressed domain were extra useful than the uncompressed domain. They suggested a deployment of their novel saliency map for new fields of application in which uncompressed domain saliency previously demonstrated its efficacy: object segmentation, video retargeting and video surveillance or discovery.

\section{System Design}

\subsection{Video Watermarking Using Spectral Centroid and Haar Wavelet Transform-Discrete Fourier Transform (HWT-DFT)}

In the proposed method, a video is given as an input and it is split into two halves, one is audio and the other is image. The audio and image watermarking process is achieved by means of utilizing two techniques. The process of audio watermarking can be done by using spectral centroid wavelet and firefly algorithm. Whereas the process of image watermarking can be achieved through discrete Fourier transform and Haar wavelet transforms.

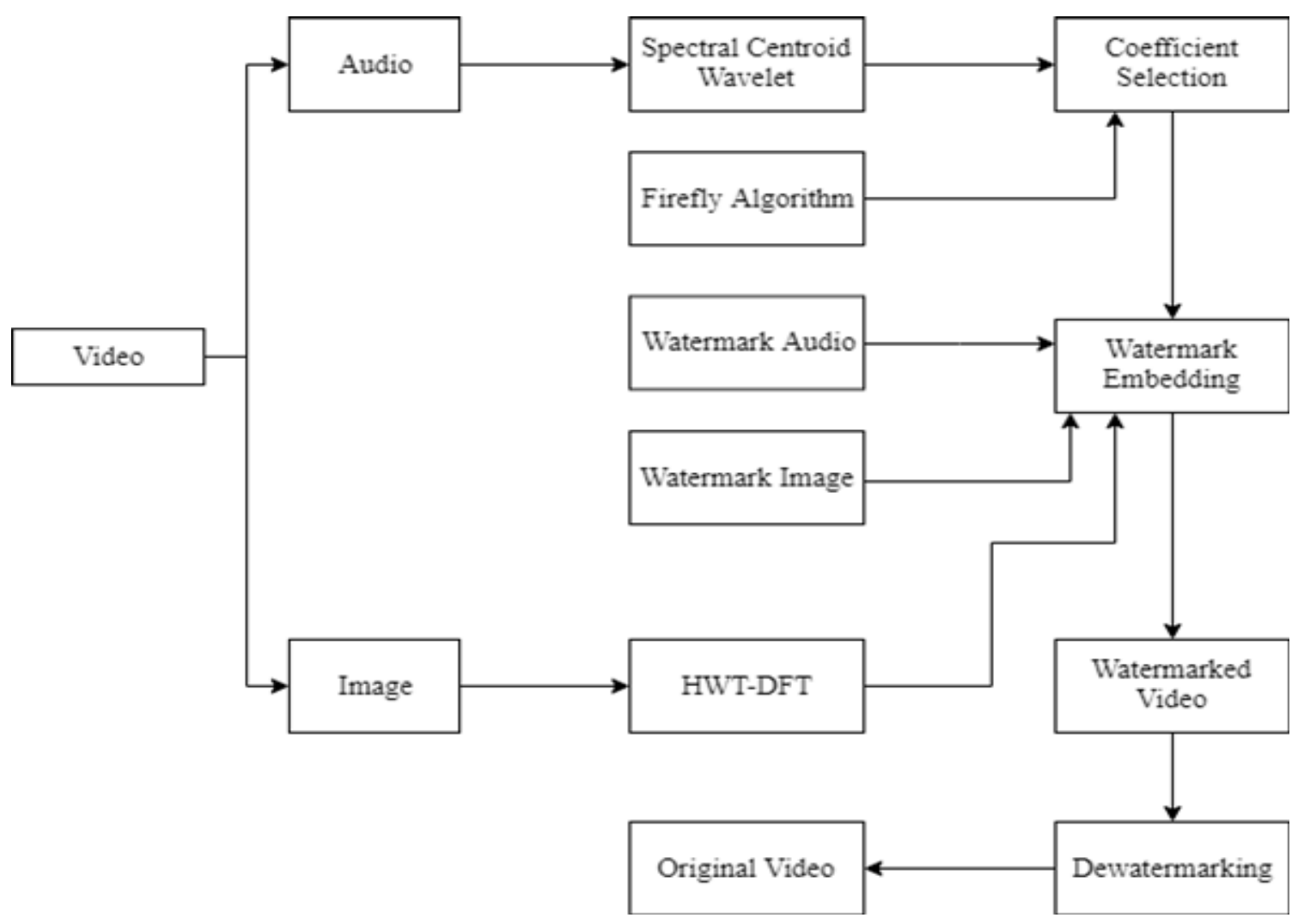

Figure 1 Watermarking Process 
Firstly, the incoming video is separated into two halves. The first half is watermarked as an audio signal whereas the second half is watermarked as an image. The above-mentioned figure 5.1 represents the chunk drawing of the future architecture. Here, the secret information is hidden as audio and an image. The security is achieved through this process.

\subsection{Audio and Image Watermarking Using HWT-DFT}

In the proposed research work, audio and image watermarking using spectral centroid wavelet and firefly algorithm. The incoming video is fragmented into two halves. The primary one is watermarked as an audio signal then the next one is watermarked as an image.

- Audio signal- Processed using Spectral Centroid Wavelet transform.

- Another audio Signal-Taken and watermarked with the input audio signal and optimized with the firefly algorithm.

- The image is processed through the Discrete Fourier Transform and compressed further by considering the high coefficient using the Haar wavelet transforms.

- Another input for image watermark is taken and combined with the previous output of image pre-processed using the embedded OOPM algorithm.

- The watermarked audio and image are combined to form watermarked video

The flow diagram of the proposed methodology is described below-given Figure 5.2. 


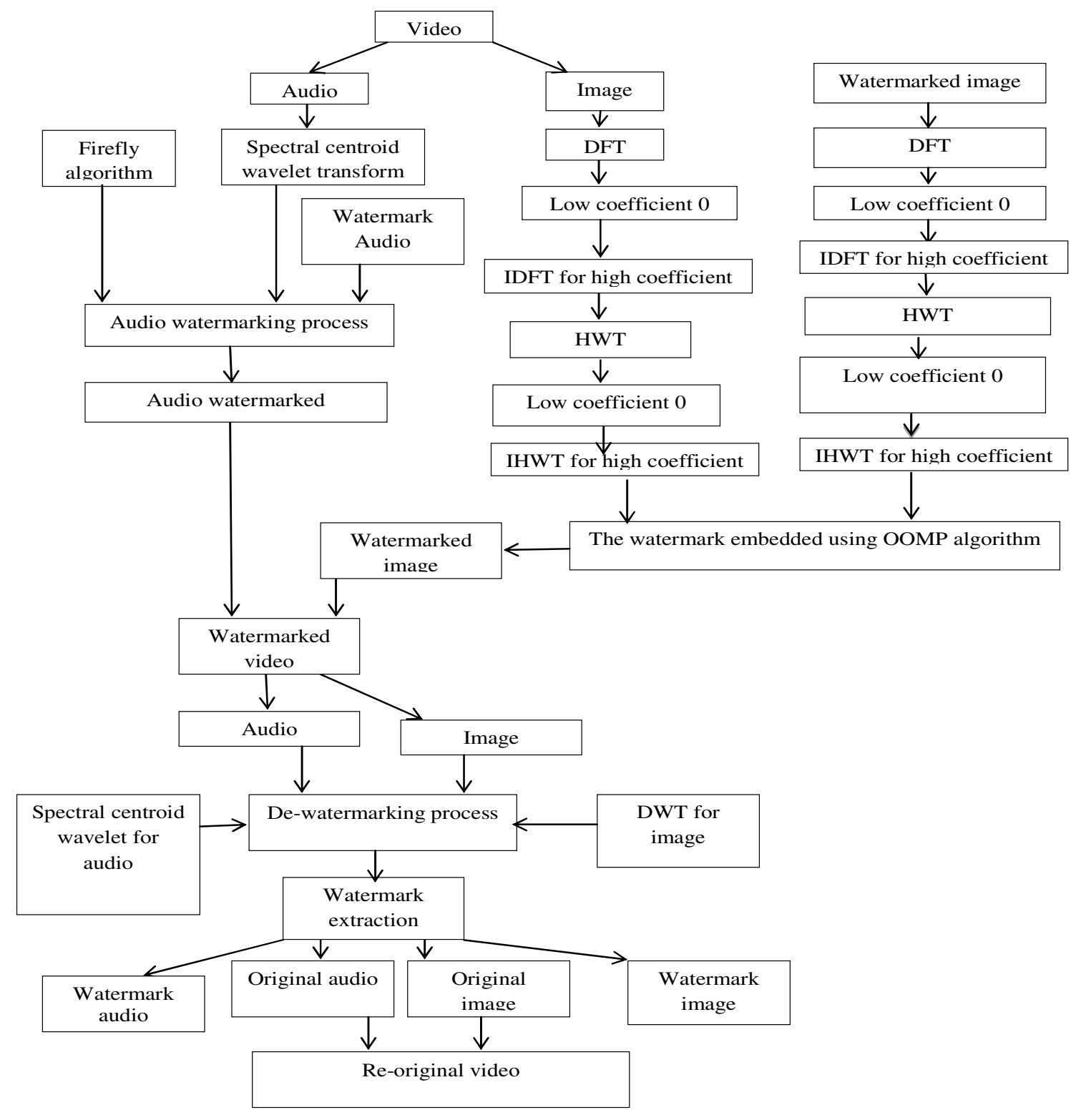

Figure 2 Flow diagram of Watermarking

\subsection{Audio Watermarking}

The audio watermarking process mainly consists of four stages namely,

- Decomposition of the signal

- Selection of Coefficients

- Generation of code and

- Implanting process 
The received audio signal is decomposed into several bands. The extraction of the feature of the audio signal is achieved through Spectral Centroid Wavelet (SCT) transform. It offers the center of gravity of the magnitude spectrum. It differs the sinusoidal wave of a signal and further defines the specific location on the sub band. Spectral Centroid Frequency (SCF) assesses the center of gravity of a spectrum in every single sub-band. SCF is computed as the average weighted frequency of the sub band as given in below Figure 5.3. The weights are the normalized energy of the corresponding frequency module. Spectral centroid defines the mass of the frequency spectrum.

$$
\mathrm{SC}=\frac{\sum_{\mathrm{k}=1}^{\mathrm{K}} \mathrm{f}_{\mathrm{k}} \mathrm{X}_{\mathrm{k}}}{\sum_{\mathrm{k}=1}^{\mathrm{K}} \mathrm{X}_{\mathrm{k}}}
$$

Where $\mathrm{X}_{\mathrm{k}}$ denotes the magnitude of the component in frequency band $\mathrm{f}_{\mathrm{k}}$.

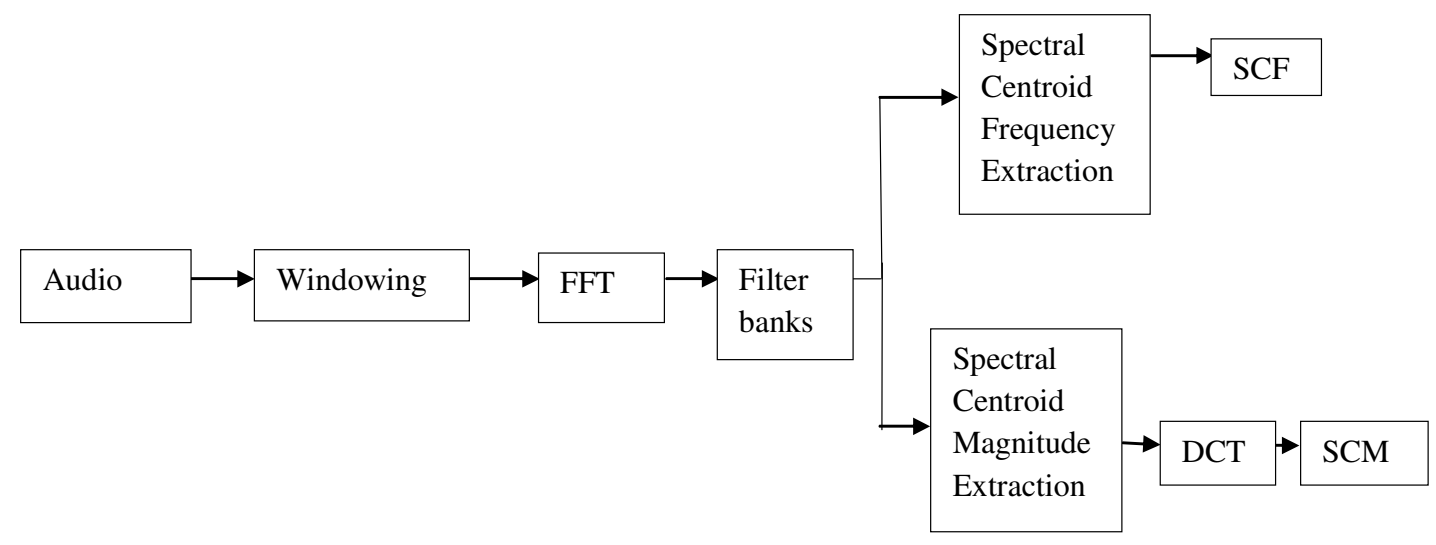

Figure 3 Block diagram of Spectral Centroid Frequency process

\subsubsection{Spectral Centroid Wavelet Transform}

The spectral centroid is the median of the spectrum. Since spectrum gives the sign of how the signal's amplitude is dispersed among the frequencies, its center of mass shows the average amount of amplitude. From the audio view, it is the average loudness, and when compared to image view, it is taken as average brightness. The weighted average is taken for calculation from the signal observed. The watermarked audio is further process through the firefly algorithm

\subsection{Firefly Algorithm (FA)}

In a FA calculation, the target work streamlining is unexpected at the brilliance notwithstanding a development of the firefly. The more brilliant firefly will bait the neighboring fireflies with low splendor. The firefly procedure starts with the firefly's populace instatement. The splendor of the firefly decides the development of the fireflies. In the iterative method, the profundity of the ithfirefly is in correlation with 
the force of jth firefly. In view of the differentiation in force, both ith firefly draw nearer to jth firefly or jth firefly will move towards ith firefly. The most appropriate arrangement got is persistently refreshed until the specific halting condition is satisfied. When the iterative framework includes an end, the agreeable answer is resolved. Figure 5.1 portrays the pseudocode of the FA calculation.

Pseudocode:

Begin;

Initialize algorithm parameters:

Define the objective function $\mathrm{f}(\mathrm{x})$, where $\mathrm{x}=(\mathrm{x} 1, \ldots \ldots . . \mathrm{xd})$

Generate initial populance of fireflies xi $(i=1,2, \ldots ., n)$

Determine the light intensity of Firefly at xi by using objective function $\mathrm{f}(\mathrm{xi})$

While (K<Maximum_Generation) // Where $\mathrm{k}=1$ to maximum

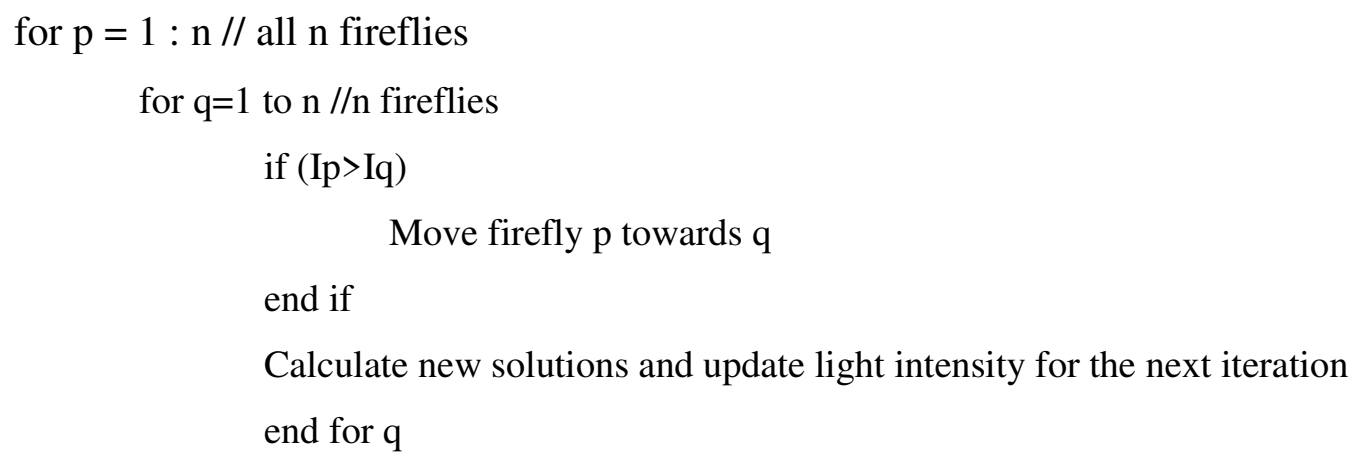

Sort the fireflies based on the intensity value and find the current best solution; end while

\subsection{Image-based Watermarking}

The image extracted from the video is pre-processed for raising the process of computation in addition to accuracy. Image extracted is processed through the DFT to acquire the feature of spatial domain of the image, for the reason that in FT image is fragmented into sinusoidal components, where it is easy to handle certain image frequencies. The features that contain higher coefficients are handled through the Inverse DFT (IDFT). The IDFT considers the complex coefficient values, in addition, to map them with its original coefficient. Supposing the original as real values in addition to the result of IDFT will be the 
complex numbers wherever the remaining coefficient is marked as zero. To improvise through the image accuracy, it is further processed through Haar wavelet transform which is similar to the Fourier transform that was utilized for the compression of an image and further multiplied with the binary matrix by means of utilizing the uniform set theory.

In the proposed method Haar wavelet is processed by considering the input from the discrete inverse transform and compresses the high-quality image which is additionally managed by means of Inverse Haar Wavelet Transform (IHWT). The output is provided to the Ordered Orthogonal Matching Pursuit (OOMP) algorithm for inserting with the watermarked image input. The new watermarked image is considered and handled by means of DFT in addition to HWT procedure as of how we process the image input from the video. The output from the video extracted image and the watermark input provides the compressed images is specified to OOMP for embedding the images.

\subsection{Haar Wavelet Transform}

Haar wavelet basis may be utilized to illustrate a picture by a wavelet rework computation. The pixel is averaged collectively pair-smart and is determined to collect the new resolution photo via pixel values. Particular records will be lost in the averaging procedure. The Haar wavelet rework is applied to investigate snap shots successfully and efficaciously at several resolutions. It is utilized to gather the approximation coefficients similarly to element coefficients at exclusive levels. The Haar remodel works like a low-skip clear out as well as a high-bypass filter out at the equal time.Haar wavelet function, represented by $\psi(\mathrm{t})$, is specified by

$$
\psi(t)=\left\{\begin{array}{l}
1,0 \leq t \leq \frac{1}{2} \\
-1, \frac{1}{2} \leq t \leq 1 \\
0, \text { elsewhere }
\end{array}\right\} z
$$

\section{Discrete Fourier transforms}

Image compression techniques such as pixel coding, predictive coding, and transform coding. The Fourier transform (FT) is a vital image processing tool founded on transform coding. The response of the conversion signifies the image in the domain of a Fourier or else frequency, even though the output image is an equivalent of a spatial domain. In an image of Fourier frequency domain, apiece point signifies a specific frequency enclosed in the image of the spatial domain. The FT is utilized in an extensive range of 
applications similar to analysis, filtering, reconstruction, and compression of an image. The 2D discrete Fourier transform (2D DFT) of an image $\mathrm{f}$, denoted by $\mathrm{F}(\mathrm{u}, \mathrm{v})$ is given by

$$
\begin{gathered}
\qquad F(u, \mathrm{v})=\sum_{x=0}^{M-1} \sum_{y=0}^{N-1} f(x, y) e^{-j 2 \pi\left(\frac{u x}{M}+\frac{\mathrm{vy}}{N}\right)} \\
\text { for } \mathrm{u}=0,1,2, \ldots \mathrm{M}-1 \text { and } \mathrm{v}=0,1,2, \ldots \mathrm{N}-1 .
\end{gathered}
$$

Given $\mathrm{F}(\mathrm{u}, \mathrm{v})$, it can obtain $\mathrm{f}(\mathrm{x}, \mathrm{y})$ back by means of the inverse DFT and given by,

$$
f(x, y)=\frac{1}{M N} \sum \sum F(\mathrm{u}, \mathrm{u}) e^{j 2 \pi\left(\frac{u x}{M}+\frac{\mathrm{vy}}{N}\right)}
$$

The values of $F(u, v)$ in the above equations are called the Fourier coefficients. For $u=0, v=0$, we have,

$$
F(0,0)=\sum_{x} \sum_{y} f(x, y)
$$

$\mathrm{f}(\mathrm{x}, \mathrm{y})$ is the entire leaden gage equal of the copy also called the dc constituent of the Fourier alter also represent zero frequency.

Even if $f(x, y)$ is real, its transform is complex. If $R(u, v)$ and $I(u, v)$ represent the real and imaginary components of $\mathrm{F}(\mathrm{u}, \mathrm{v})$, the DFT can be expressed in polar form,

$$
F(u, v)=|F(u, v)| e^{j \emptyset(u, v)}
$$

Where

Magnitude $|F(u, v)|=\sqrt{R^{2}(u, v)+I^{2}((u, v)}$ is called the Fourier spectrum and

$\emptyset(u, v)=\tan ^{-1}\left(\frac{I(u, v)}{R(u, v)}\right)$ is the phase angle

If $\mathrm{f}(\mathrm{x}, \mathrm{y})$ is real, its Fourier transform is conjugate symmetric about the origin, i.e.,

$\mathrm{F}(\mathrm{u}, \mathrm{v})=\mathrm{F}^{*}(-\mathrm{u},-\mathrm{v})$ which implies that the Fourier spectrum is also symmetric about the origin, i.e.,

$$
F(u, v)|=| F(-u,-v) \mid
$$

Also, $F(u, v)=F(u+M, v)=F(u, v+N)=F(u+M, v+N)$, implies that the DFT is infinitely periodic in both $\mathrm{u}$ and $\mathrm{v}$ directions, with the periodicity determined by $\mathrm{M}$ and $\mathrm{N}$. 
Periodicity is also a property of the inverse DFT,

$$
f(x, y)=f(x+M, y)=f(x, y+N)=f(x+M, y+N)
$$

Thus, an image obtained by taking the inverse Fourier transform is also infinitely periodic. But DFT implementations compute only one period, so we work with arrays of size $\mathrm{M} \times \mathrm{N}$.

The Fourier transform pair satisfies the following translation properties,

$$
f(\mathrm{x}, \mathrm{y}) e^{j 2 \pi\left(\frac{u_{0} x}{M}+\frac{v_{0}}{N}\right)}<=>F\left(u-u_{0}, v-v_{0}\right)
$$

And $\quad f\left(x-x_{0}, \mathrm{y}-\mathrm{y}_{0}\right)<=>F(u, v) e^{-j 2 \pi\left(\frac{x_{0} u}{M}+\frac{\mathrm{y}_{0} \mathrm{v}}{N}\right)}$

That is multiplying $\mathrm{f}(\mathrm{x}, \mathrm{y})$ by the exponential shifts the origin of the DFT to $\left(u_{0}=\frac{M}{2}, \mathrm{v}_{0}=\frac{N}{2}\right)$ and conversely, multiplying $F(u, v)$ by the negative of that exponential shifts the origin of $f(x, y)$ to $\left(x_{0}, y_{0}\right)$.

$$
\text { For } u_{0}=\frac{M}{2}, v_{0}=\frac{N}{2} \text {, }
$$

we have

$$
f(x, y)(-1)^{x+y}<=>F\left(u-\frac{M}{2}, v-\frac{N}{2}\right)
$$

The input image multiply by $(-1)^{x+y}$ prior to computing $(\mathrm{u}, \mathrm{v})$ in image processing is common and it shifts the data so that $\mathrm{F}(0,0)$ is at the center of the frequency rectangle defined by the intervals $[0, \mathrm{M}-1]$ and $[0, \mathrm{~N}-1]$.

\subsection{Ordered Orthogonal Matching Pursuit Algorithm}

The OOMP is enhanced version of OMP procedure that notices less co-efficient in the support set is removed by associating with the projection coefficients. The procedure is scaled to evade re-entry. It achieves accurate retrieval of copy with the less quantity of measurements. Furthermore, the optimum values are transformed into binary signals for less computation of the complete image. Thus produces an embedded output of a watermarked image by means of associating input images coefficient.

\subsection{De-Watermarking Process}

The image output is united with a watermarked audio to produce a watermarked video. At any time if the fusion process happens, it should also be competent to acquire back the original copy. The procedure of attaining the original copy back by way of eliminating the watermark from the video is known as the De 
watermarking process. This procedure pursues a similar process which was followed by the watermarking process, but the only difference or dissimilarity is that this process can be implied with the different algorithms. The watermarked video is separated for the second time into audio and image. Audio is dewatermarked through the spectral centroid wavelet transform, and the Discrete Wavelet Transform remarks the image. Subsequently the removal of a watermark, the audio and image are additionally merged to produce the original copy of the video.

\subsection{Audio De-Watermarking}

Sound sign $A *$ which is going to be removed is separated into $M$ sound casings, indicated as $A * 1(p), p=$ $1,2, \ldots, M$. At that point M1 sound sub-groups are acquired from every sound casing, signified as $A * 2(p$, q), $\mathrm{q}=1,2, \ldots, \mathrm{M} 1$.

The centroid $\mathrm{C} *(\mathrm{p})$ of every sound casing is processed, at that point the remade watermark $\mathrm{W} *$ is gotten on sound sub-band $A * 2(p, q c)$ conveying centroid of sound casing, and acquired the coefficients meant as G*pqc. Removing watermark bits from the front $\mathrm{n} / \mathrm{n} 1$ space coefficients, and the principles are appeared as follows: $w^{\prime}(i)=\left\{\begin{array}{l}0,\left[G * \frac{p q c}{\Delta}\right] \bmod 2=0, \\ 1,\left[G * \frac{p q c}{\Delta}\right] \bmod 2=1 .\end{array}\right.$

Hence the extracted watermark is $\mathrm{W}=\{\mathrm{w}(\mathrm{i}) \in\{0,1\} \mid \mathrm{i}=1,2, \ldots, \mathrm{M} \times(\mathrm{n} / \mathrm{n} 1)\}$.

\subsection{Image De-Watermarking}

In this procedure right off the bat DWT is applied to watermarked picture and spread picture which deteriorated the picture in sub-groups. After that the watermark is recuperated from the watermarked picture by utilizing the equation of the alpha mixing.

As per the recipe of the alpha mixing the recuperated picture is given by

$$
\mathrm{RW}=(\mathrm{WMI}-\mathrm{k} * \mathrm{LL})
$$

Where RW= Low recurrence estimate of Recovered watermark, LL= Low recurrence guess of the first picture, and WMI= Low recurrence estimate of watermarked picture. The underlying worth is executed tasks like the installing of watermark. After extraction process, Inverse discrete wavelet transform is applied to the watermark image coefficient to generate the final watermark extracted image

In the extraction phase, the watermark photo is retrieved from the watermarked picture with the use of inverse DWT. Initially, Bi-orthogonal transform primarily based IDWT is employed, the pleasant band 
and location are selected as discussed earlier. Selection of band is primarily based on the entropy wherein the band having most entropy is selected. Selection of region is based at the importance version wherein the places with maximum Importance Measures are taken. Due to the entropy parameter geometric distortion like translation, shearing, cropping, alternate of element ratio etc. Can forget the lack of geometric synchronization during de watermark process.5 Results of Watermarking Using HWT-DFT.

This section discusses the results obtained from image and audio watermarking processes which are described below.

\section{Image Watermarking}

\subsection{Image watermarking process}

The quality of the original signal is identified by this parameter. It gives information about the similarity between the original and the watermarked audio signal. PSNR is calculated by using

$$
P S N R=20 \log 10 \frac{255}{\sqrt{M S E}}
$$

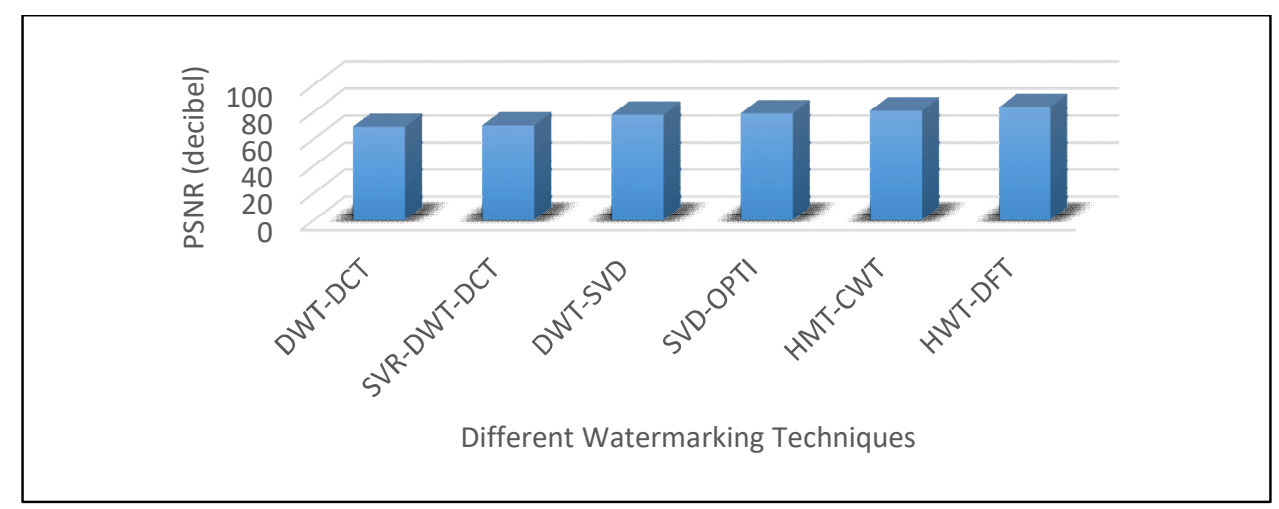

Figure 4 PSNR of Images

The method which has the highest PSNR value gives overall similarity between the original and watermarked image. Different attacks give different PSNR values.

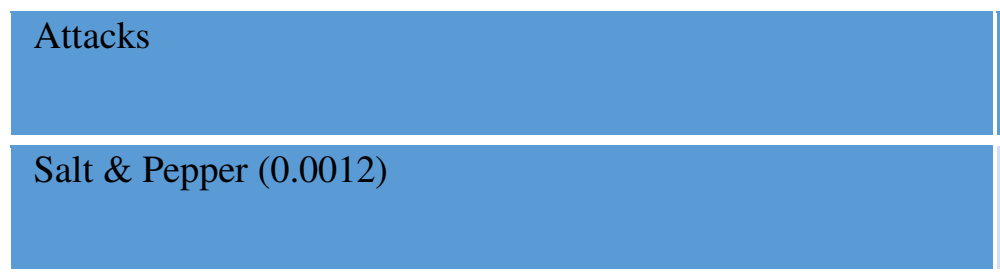

\section{PSNR}

48.4371 


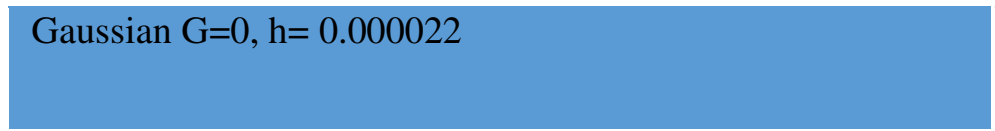

Figure 5 Performance of PSNR based on attacks

\subsection{Normalized correlation (NC)}

Normalized correlation checks the similarity between the original image and watermarked image and audio signal. NC is determined by

$$
N C=\frac{\sum_{i=1}^{M} \sum_{j=1}^{N}\left(f(i, j) f^{\prime}(i, j)\right)^{2}}{\sum_{i=1}^{M} \sum_{j=1}^{N}(f[i, j])^{2}}
$$

Where $F(i, j)$ - represents unique image,

$F(i, j)$ - represents the waterlined image,

M- Stature,

N- Thickness

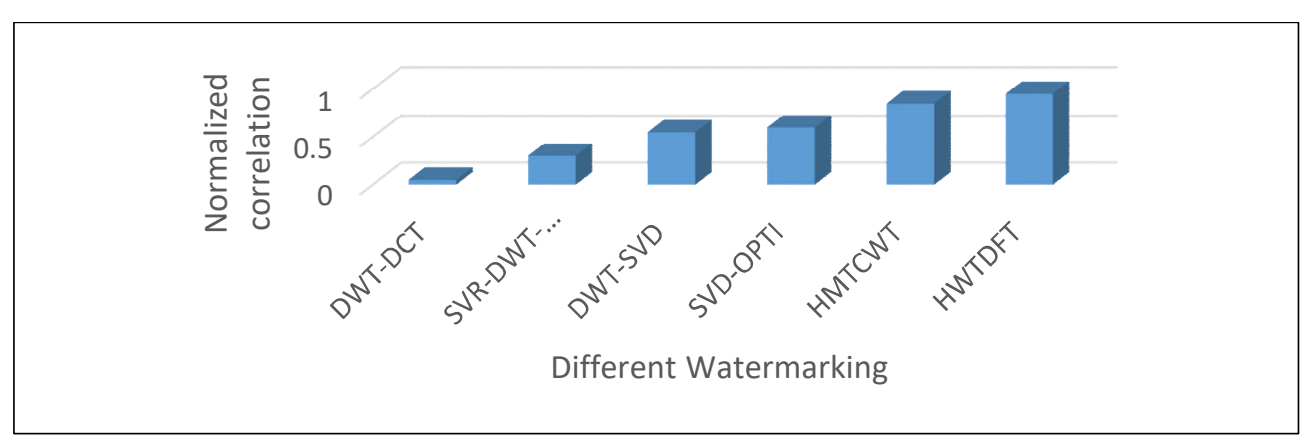

Figure 6 Normalized Correlation (NC)

\subsection{Mean square Error (MSE)}

MSE represents the average of square error. It calculates the error amount that differs by the pixel assessment of the unique image from a watermarked picture. HWTDFT consumes minimum error rate as the watermarked image is similar to the original image. It provides high security. 


$$
M S E=\frac{1}{M N} \sum_{i=1}^{M} \sum_{j=1}^{N}\left(f(i, j)-f^{\prime}(i, j)\right)^{2}
$$

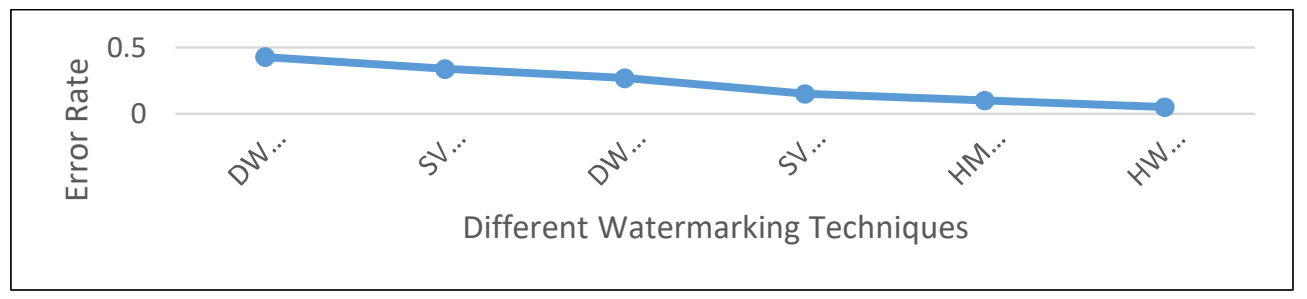

Figure 7 Mean Square Error

\subsection{Accuracy}

HWTDFT provides high accuracy. It gives about $95.1 \%$ of accuracy. Thus it is used to provide high security without losing the original image. The future system delivers the safety to the material professionally with the least fault amount .When compared to the existing methods, the proposed system has achieved the highest accuracy

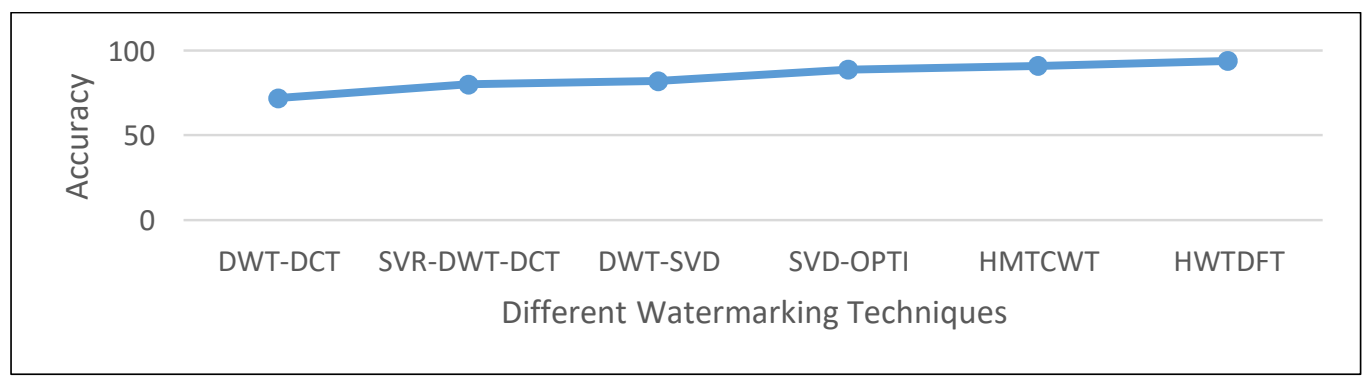

Figure 8 ACCURACY

Structural Similarity Index (SSIM), PSNR, NC, and MSE, for determining the quality of an image is calculated from video 1, 2, 3, 4 and 5. PSNR values obtained using this technique is high when compared. About $84 \%$ of PSNR is obtained. Mean Square Error obtained is very less. So, a secured image can be obtained. MSE obtained is about 0.003 .

In video 1 , image 1 is the original image and image 2 is the secret image to be watermarked. Watermarked image is the last in which the secret image is watermarked in image 1.

Table 1 Videos showing the images, watermark and watermarked images 


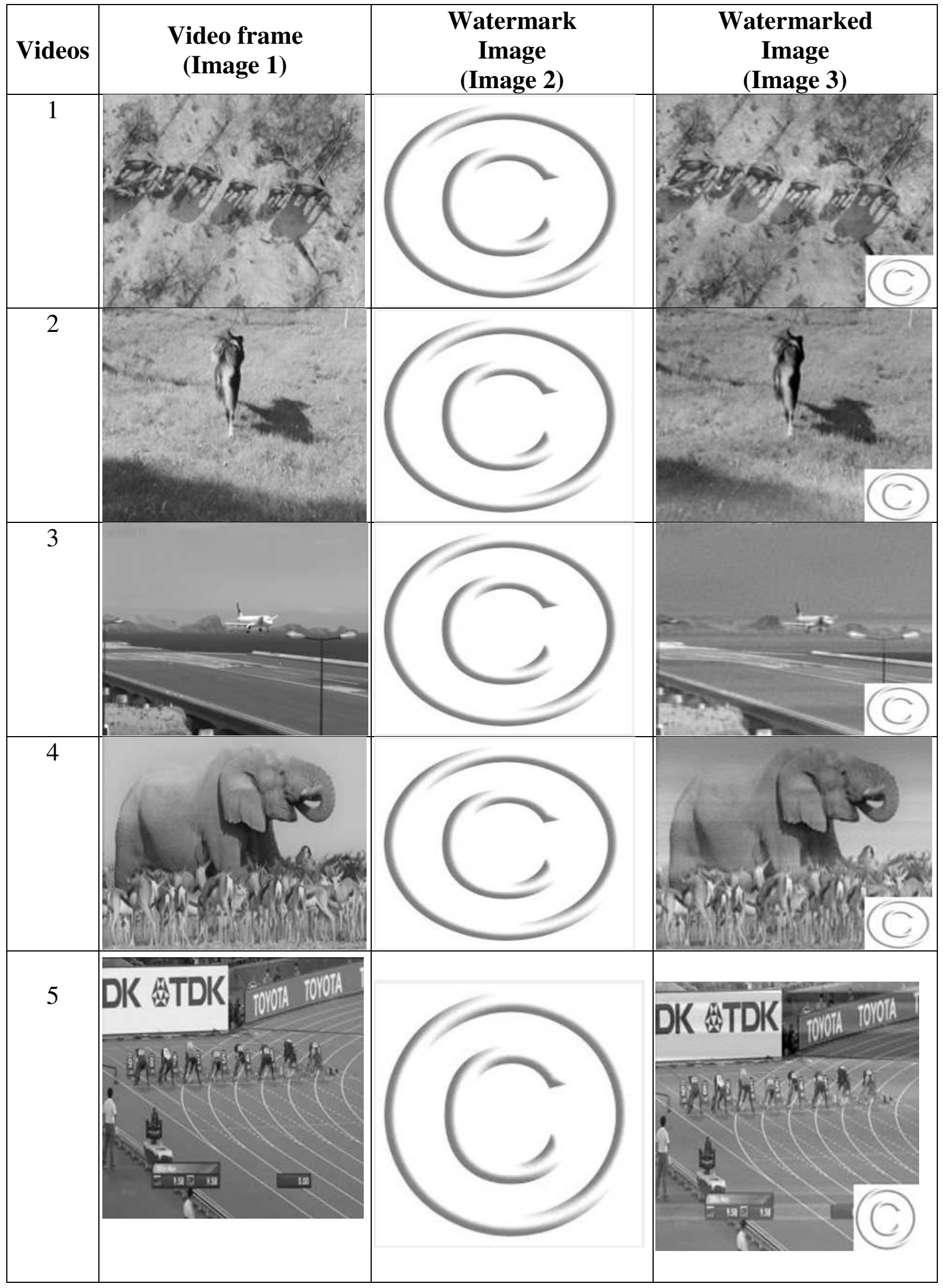


In video 2 , picture 1 is the first picture and picture 2 is the mystery picture to be watermarked. The last picture is a watermarked picture in which the mystery picture is watermarked in picture 1.

In video 3, picture 1 is the first picture and picture 2 is the mystery picture to be watermarked. The last picture is the watermarked picture in which the mystery picture is watermarked in picture 1.

In video 4 , picture 1 is the first picture and picture 2 is the mystery picture to be watermarked. The last picture is the watermarked picture in which the mystery picture is watermarked in picture 1.

In video 5 , picture 1 is the first picture and picture 2 is the mystery picture to be watermarked. The last picture is the watermarked picture in which the mystery picture is watermarked in picture 1.

Table 2 Performance evaluation using HWT-DFT

\begin{tabular}{|c|c|c|c|c|c|c|}
\hline INPUT & $\begin{array}{l}\text { Length } \\
\text { video } \\
\text { Seconds) }\end{array}$ & $\begin{array}{l}\text { of } \\
\text { in } \\
\text { frames }\end{array}$ & ${ }^{\text {of }}$ PSNR & MSE & $\mathrm{NCC}$ & SSIM \\
\hline Video1 & 18 & 445 & 64.63 & 0.022 & 0.852 & 0.641 \\
\hline Video2 & 18 & 445 & 59.34 & 0.075 & 1.3 & 0.482 \\
\hline Video3 & 12 & 311 & 68.31 & 0.009 & 0.964 & 0.679 \\
\hline Video4 & 18 & 445 & 58.56 & 0.09 & 2.101 & 0.482 \\
\hline Video5 & 22 & 550 & 64.73 & 0.021 & 1.346 & 0.708 \\
\hline
\end{tabular}

\subsection{Mean-Squared Error (MSE)}

The MSE represents the cumulative squared error between the watermarked image and the original image

$$
M S E=\frac{\sum_{M, N}\left[I_{1}(m, n)-I_{2}(M, N)\right]^{2}}{M * N}
$$

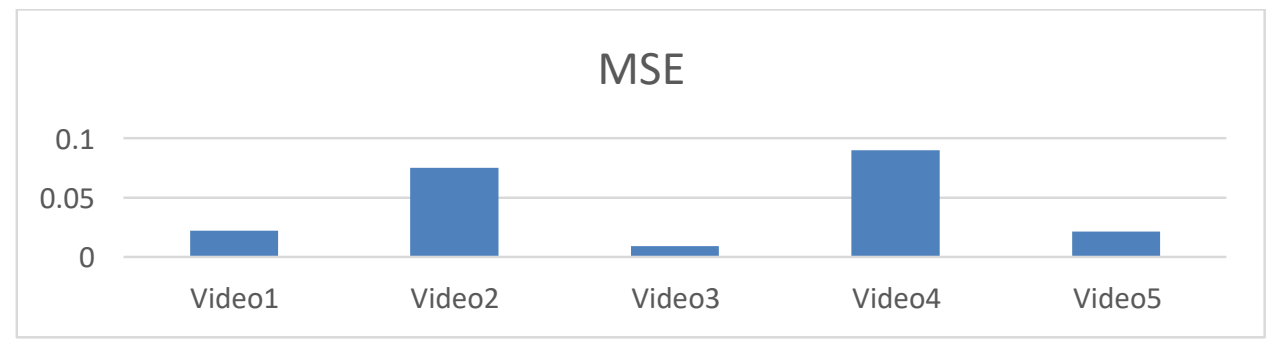




\section{Figure 9 MSE of Images}

As shown in Figure 5.4, the Mean Square Error of different images in videos is shown. The MSE is higher for video 1 when compared with video 2 and video 3. Video 4 MSE value is somewhat more as compared to video 2, and video 5 MSE value is less than video four but more than that of video 3.

\subsection{Peak Signal-To-Noise Ratio (PSNR)}

The pinnacle signal-to-clamor proportion, in decibels, between two pictures. This proportion is frequently utilized as a quality estimation between the first and a watermarked picture. The higher the PSNR, the better the quality picture. To register the PSNR, the square initially figures the mean-squared blunder utilizing the previously mentioned Equation (5.12):

In the past condition, $\mathrm{M}$ and $\mathrm{N}$ are the quantity of lines and sections in the info pictures, individually. At that point the square figures the PSNR utilizing the accompanying condition:

$$
. P S N R=10 \log _{10}\left(\frac{R^{2}}{M S E}\right)
$$

In the past condition, $\mathrm{R}$ is the greatest variance in the information picture information type. For instance, on the off chance that the info picture has a twofold exactness drifting point information type, at that point $\mathrm{R}$ is 1 . On the off chance that it has a 8-piece unsigned whole number information type, $\mathrm{R}$ is 255 , and so forth.

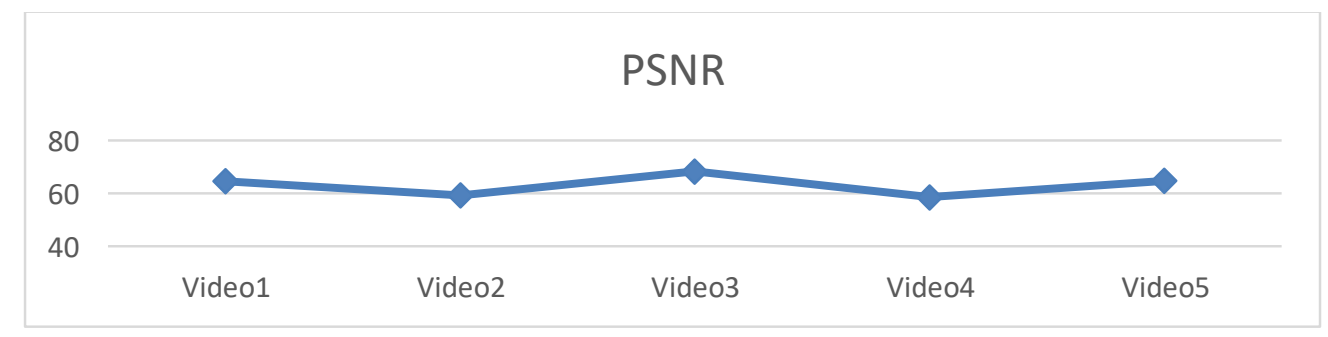

Figure 10 PSNR of Images

Figure 5.5 shows the PSNR values of images. The PSNR value is higher for video 1 . Video 2 has the lower PSNR value. Video 3 PSNR value is more compared to all the five videos. Video 4 has the PSNR valueless compared to all the videos PSNR values. Video 5 has more PSNR compared to video 2 and 4.

\subsection{Normalized Cross Correlation (NCC)}

The $N C C$ gets the values in the interval $[0,1]$, where 1 indicates the best match. 


$$
N C C=\frac{\sum_{i=1}^{M N} x_{i} y_{i}}{\sqrt{\sum_{i=1}^{M N} x_{i}^{2} \sum_{i=1}^{M N} y_{i}^{2}}}
$$

Figure 5.6 shows the Normalized Coefficient of images. Video 1 NCC value is less compared to all the videos. Video 2 is having more $\mathrm{NC}$ value compared to video 1 and 3. Video $4 \mathrm{NC}$ is more compared to all the four videos.

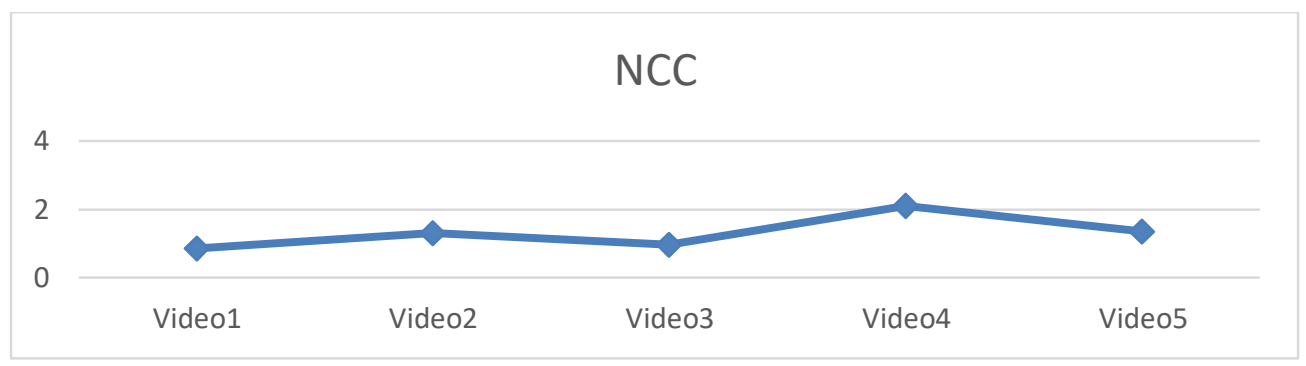

Figure 11 Normalized Coefficients of Images

\subsection{Structural Similarity Index(SSIM)}

Structural Similarity Index (SSIM) for measuring image quality.

$$
\operatorname{SSIM}(x, y)=\frac{\left(2 \mu_{x} \mu_{y}+C_{1}\right)\left(2 \sigma_{x y}+C_{2}\right)}{\left(\mu_{x}^{2}+\mu_{y}^{2}+C_{1}\right)\left(\sigma_{x}^{2}+\sigma_{y}^{2}+C_{2}\right)}
$$

where $\mu_{\mathrm{x}}, \mu_{\mathrm{y}}, \sigma_{\mathrm{x}}, \sigma_{\mathrm{y}}$, and $\sigma_{\mathrm{xy}}$ are the local means, standard deviations, and cross-covariance for images $x, y$. If $\alpha=\beta=\gamma=1$ (the default for Exponents), and $\mathrm{C}_{3}=\mathrm{C}_{2} / 2$ (default selection of $\mathrm{C} 3$ ) the index simplifies.

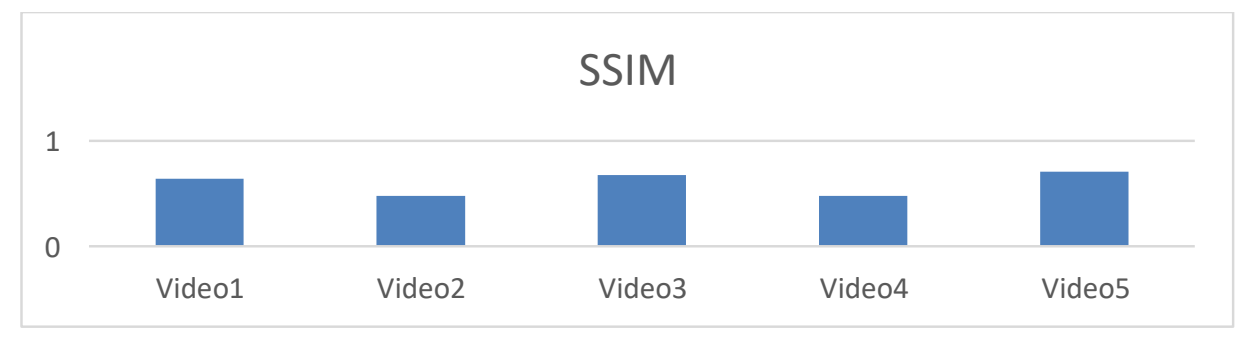

\section{Figure 12 SSIM of Images}

Figure 5.7 shows the Structural Similarity Index of all the five videos. Video 5 SSIM is very more compared to other videos. Video 2 and 4 SSIM value is almost identical.

\subsection{Audio Watermarking results}




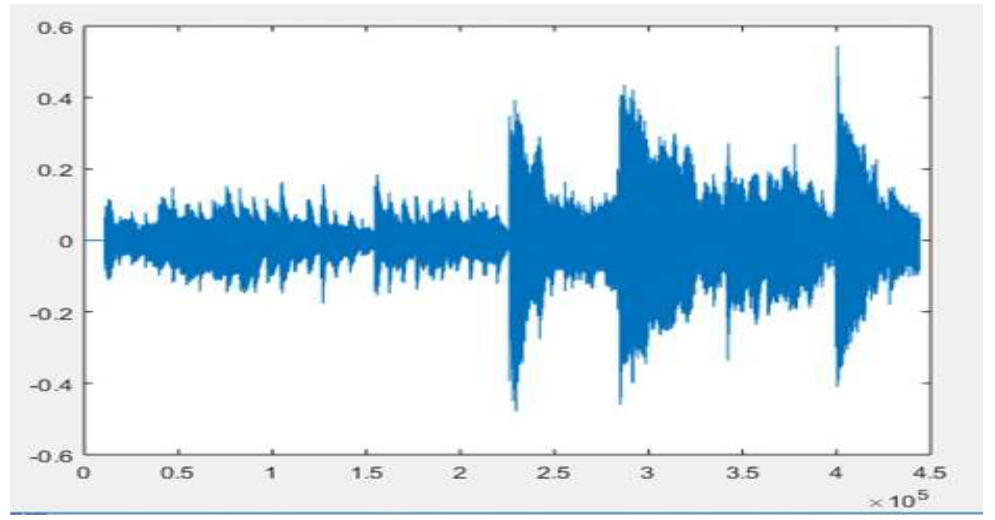

Figure 13 Input Audio signal

Shows the input audio signal. The input is the audio signal given to follow the performance.

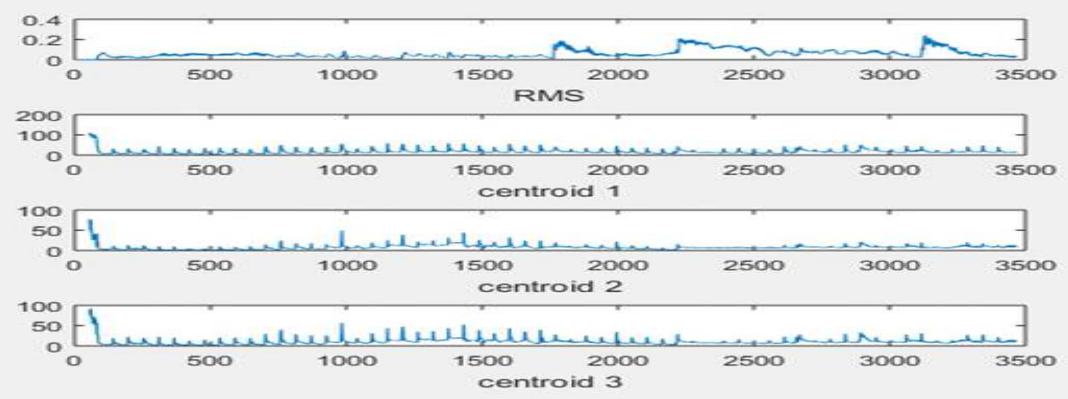

Figure 14 Graph of Audio signal using Spectral Centroid.

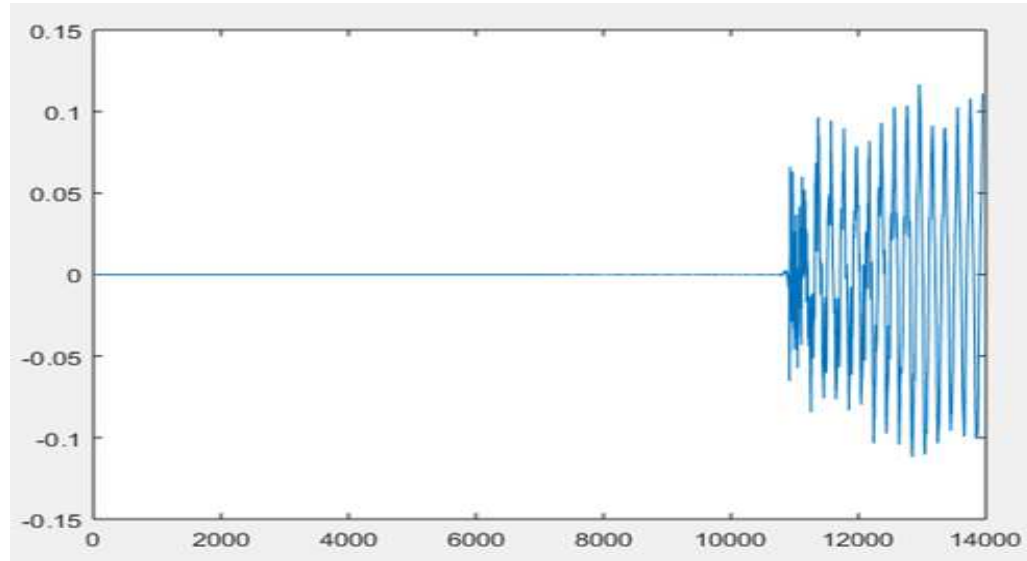

Figure 15 Attack

Shows the attack in the noise signal. Figure 15.

shows the watermarked audio signal. The audio signal given as input is watermarked. 


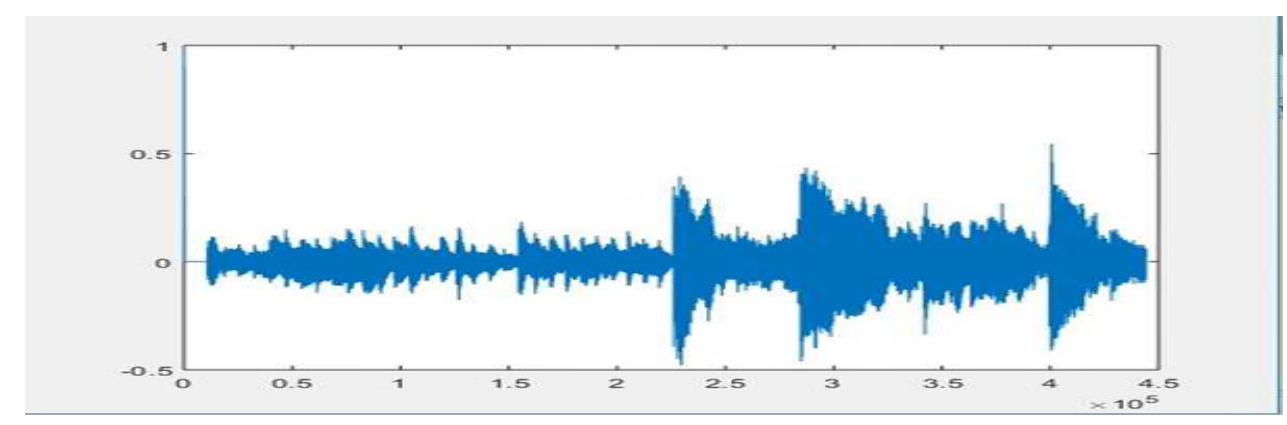

Figure 16 Watermarked audio

The images are handled through HWT-DFT. Then the output watermarked images and audio combined together to form a watermarked video. The obtained video is de-watermarked to form the original copy of a video. The procedure of getting back the original copy by means of eliminating the watermark from the video is known as as de-watermarking. The below table 4 shows the watermarked Image, copyright image, reconstructed video frame after the process of De- Watermarking.

Table 3Table showing the Watermarked Image, copyright image and reconstructed video frame after Dewatermarking process

\begin{tabular}{|c|c|c|c|}
\hline $\begin{array}{c}\text { Watermarked } \\
\text { Videos no. }\end{array}$ & $\begin{array}{c}\text { Watermarked Image } \\
\text { (Image 1) }\end{array}$ & $\begin{array}{c}\text { Copyright image } \\
\text { (Image 2) }\end{array}$ & $\begin{array}{c}\text { Reconstructed video frame } \\
\text { after Dewatermarking } \\
\text { (Image 3) }\end{array}$ \\
\hline 1 & & & \\
& & & \\
\hline
\end{tabular}




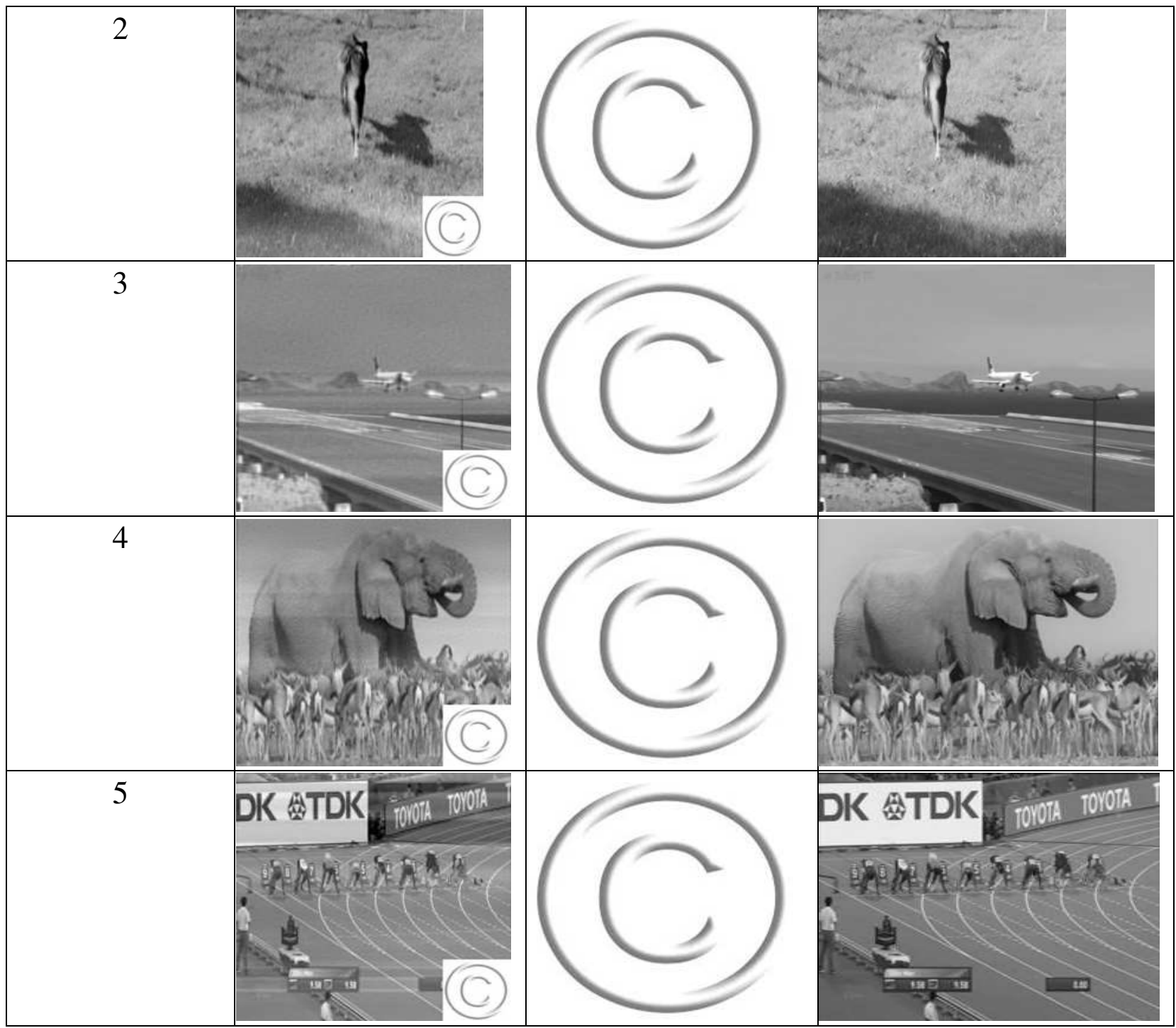

The comparison of the computational complexity is given in the Table 4.4. Two existing algorithms are mentioned in the table namely, Symmetric local binary pattern, and LWT-SVD algorithm. The proposed algorithm was found to perform the watermarking technique with lesser computational complexity as compared to the existing algorithms.

Table 4 Comparison of Computational Complexity

\begin{tabular}{|l|l|}
\hline Methods & Time (Secs) \\
\hline $\begin{array}{l}\text { Symmetric local binary pattern (Tuncer, } \\
\text { T \& Kaya, M., 2019) }\end{array}$ & 9.37 \\
\hline Proposed methodology & 3.13 \\
\hline
\end{tabular}




\section{Conclusion}

In this chapter, Video will be given as a input that is divided into two parts that is audio and image. The process of audio and image watermarking using HWT-DFT was explained. The audio was processed through Spectral Centroid Wavelet Transform and optimized by using Firefly Algorithm. The image was handled through HWT and DFT. Another input for image watermark is taken and combined with the previous output of image pre-processed using the embedded OOPM algorithm. The watermarked audio and image are combined to form watermarked video. SSIM, PSNR, NC, and MSE parameters considered for determining the quality of an image which was calculated from video 1, 2, 3, 4 and 5 as mentioned in the above sections and De-watermarking process can be applied to retrieve the original video.

The HWTDFT delivers the safety to the information without behind the innovation of the image. By using HWTDFT, the PSNR achieved was 84.09, DWT-DCT (64.71), DCT- DWT (65.11), DWT- SVD (71.32), and SVD- OPTI (73.19). The method which has the highest PSNR value gives overall resemblance between the unique and waterlined image. Different bouts give different PSNR values. NC is greater for HWTDFT technique when compared to other techniques. HWTDFT consumes minimum error rate as the watermarked image is like to the unique picture. It provides high security. HWT-DFT provides high quality of audio signal and Spectral centroid frequency and Spectral centroid Magnitude are calculated.

\subsection{Conclusion And Future Scope}

HWT-DFT technique, video might be given as input and its miles divided in to components audio and photograph. The Audio is processed via Spectral Centroid Wavelet Transform and optimized by the use of Firefly Algorithm. The photograph is processed thru Haar Wavelet Transform further to Discrete Fourier Transform.

The efficiency of the gadget is evaluated with the assist of the PSNR, NC, error charge and the accuracy of the security degree in HWT-DFT. For this evaluation, 4 existing strategies are considered which might be namely, DWT-DCT, SVR- DCT- DWT, DWT- SVD, and SVD- OPTI. Through these four existing strategies the proposed approach 1 HWT-DFT method is in comparison for one of a kind metrics, and its performance is analyzed. The HWTDFT delivers the safety to the records without dropping the innovation of the copy. By the usage of HWTDFT, the PSNR carried out was 84.09, DWT-DCT (64.71), DCT- DWT (65.11), DWT- SVD (71.32), and SVD- OPTI (73.19).

The method which has the highest PSNR value gives overall resemblance amid the unique and waterlined copy. Different bouts give different PSNR values. NC is greater for HWTDFT technique when compared 
to other techniques. HWTDFT consumes minimum error rate as the watermarked image is like to the original picture. It provides high security.

In HWT-DFT, the results are analyzed for PSNR, MSE, NC, and Structural Similarity Index for Measuring image quality (SSIM) is calculated for frame 100, frame 150 and frame 200. PSNR values obtained using this technique is high when compared to other existing techniques it is observed as $86 \%$ of PSNR. Mean Square Error obtained is very less. So, a secured image can be obtained. MSE obtained is about 0.003. The MSE is higher for frame 150 when compared with frame 100 and frame 200. The PSNR value is higher for frame 100. Frame 150 has the lower PSNR value.

\section{FUTURE SCOPE}

The future directions for this study will be as follows:

\section{1) Removal of Multiple types of Noise}

An image may be corrupted by multiple types of noise. Image fusion algorithms can be implemented so that multiple types of noise can be removed.

\section{2) Fusion in the presence of blur}

Presence of blur is another common problem of imaging application. Presence of blur in the image makes the extraction of the critical feature, difficult. Blur may occur simultaneously with different types of noise. Hence, deblur is also an important task to increase the information content and quality of an image. Therefore, deblurring should also be incorporated with image fusion both in the presence or absence of noise to increase the adaptiveness of the fusion algorithm.

\section{3) Image Registration}

In the future, work could be done on image registration, and it should be combined with the image fusion. So that, the fusion algorithms would not be limited to the registered images and can be applied to unregistered images.

\section{4) Hybrid Algorithm}

Hybrid algorithm (FA) and differential evolution (DE) called as Hybrid Firefly Algorithm (HFA) that combines the attraction mechanism of FA with the mixing ability of DE which increases the speed of convergence and the population diversity. 
[1] AbdelhamidBenhocine, LamriLaouamer, Laurent Tchamnda Nana, Anca Christine Pascu Improving extraction of watermarks in color attacked watermarked images Journal of Communication and Computer, ISSN 1548-7709, USA

[2] Abraham, J., \& Paul, V. (2016). An imperceptible spatial domain color image watermarking scheme. Journal of King Saud University - Computer and Information Sciences. doi:10.1016/j.jksuci.2016.12.004

[3] Abuturab, M. R. (2017). Multiple color-image fusion and watermarking based on optical interference and wavelet transform. Optics and Lasers in Engineering, 89, 47-58. doi:10.1016/j.optlaseng.2016.02.014

[4] Ahmad, F., \& Cheng, L.-M. (2018). Authenticity and copyright verification of printed images. Signal Processing, 148, 322-335. doi:10.1016/j.sigpro.2018.02.029

[5] Albalawi, U., Mohanty, S. P., \&Kougianos, E. (2017). A new region aware invisible robust blind watermarking approach. Multimedia Tools and Applications, 76(20), 21303-21337.

[6] Ammar, M., Mitrea, M., Hasnaoui, M., \& Le Callet, P. (2018). MPEG-4 AVC stream-based saliency detection. Application to robust watermarking. Signal Processing: Image Communication, 60, 116-130. doi:10.1016/j.image.2017.09.007

[7] Ancuti, Codruta O.; Ancuti, Cosmin; De Vleeschouwer, Christophe; Bovik, Alan C.. Single-Scale Fusion: An Effective Approach to Merging Images. In: IEEE Transactions on Image Processing, Vol. 26, no.1, p. 65-78 (2017)

[8] Ansari, A., Hong, S., Saavedra, G., Javidi, B., \& Martinez-Corral, M. (2018). Ownership protection of plenoptic images by robust and reversible watermarking. Optics and Lasers in Engineering, 107, 325-334. doi:10.1016/j.optlaseng.2018.03.028

[9] Araghi, T. K., Manaf, A. A., \&Araghi, S. K. (2018). A secure blind discrete wavelet transform based watermarking scheme using two-level singular value decomposition. Expert Systems with Applications, 112, 208-228. doi:10.1016/j.eswa.2018.06.024

[10] Asikuzzaman, M., \& Pickering, M. R. (2017). An Overview of Digital Video Watermarking. IEEE Transactions on Circuits and Systems for Video Technology, 1-1. doi:10.1109/tcsvt.2017.2712162

[11] Bai, Y., Bai, S., Zhu, G., You, C., \& Liu, B. (2010, October). A blind audio watermarking algorithm based on FFT coefficients quantization. In Artificial Intelligence and Education (ICAIE), 2010 International Conference on (pp. 529-533). IEEE.

[12] Barni, M., \&Bartolini, F. (Eds.). (2004). Watermarking systems engineering: enabling digital assets security and other applications. CRC Press.

[13] Barni, M., Bartolini, F., Caldelli, R., De Rosa, A., \&Piva, A. (2000, May). A robust watermarking approach for raw video. In Proceedings of the 10th international packet video workshop.

[14] Bassali, H. S., Chhugani, J., Agarwal, S., Aggarwal, A., \&Dubey, P. (2000). Compression tolerant watermarking for image verification. In Image Processing, 2000. Proceedings. 2000 International Conference on (Vol. 1, pp. 430-433). IEEE. 
[15] Behnia, S., Yahyavi, M., \&Habibpourbisafar, R. (2017). Watermarking based on discrete wavelet transform and $\mathrm{q}$-deformed chaotic map. Chaos, Solitons\& Fractals, 104, 6-17. doi:10.1016/j.chaos.2017.07.020

[16] Bender, W., Gruhl, D., Morimoto, N., \& Lu, A. (1996). Techniques for data hiding. IBM systems journal, 35(3.4), 313-336.

[17] Bhagat, A., \& Singh, S. (2014). Digital Image Watermarking Technique. International Journal Of Engineering And Computer Science, 3(07).

[18] Boney, L., Tewfik, A. H., \&Hamdy, K. N. (1996, June). Digital watermarks for audio signals. In Multimedia Computing and Systems, 1996.,Proceedings of the Third IEEE International Conference on (pp. 473-480). IEEE.

[19] Boopathi, A. M., \&Abudhahir, A. (2015). Firefly algorithm tuned fuzzy set-point weighted PID controller for antilock braking systems. Journal of Engineering Research, 3(2).

[20] Bravo-Solorio, S., Calderon, F., Li, C.-T., \& Nandi, A. K. (2018). Fast, fragile watermark embedding and iterative mechanism with high self-restoration performance. Digital Signal Processing, 73, 83-92. doi:10.1016/j.dsp.2017.11.005

[21] Brindha, N. V., \&Meenakshi, V. S. (2018). Comparison Analysis of Biowatermarking Using DWT, DCT and LSB Algorithms. Lecture Notes in Computational Vision and Biomechanics, 849-863. doi:10.1007/978-3-319-71767-8_73

[22] Buckley, M. E., Ramos, M. G., Hemami, S. S., \& Wicker, S. B. (2000, September). Perceptuallybased robust image transmission over wireless channels. In Image Processing, 2000. Proceedings. 2000 International Conference on (Vol. 2, pp. 128-131). IEEE.

[23] Cao, F., An, B., Wang, J., Ye, D., \& Wang, H. (2017). Hierarchical recovery for tampered images based on watermark self-embedding. Displays, 46, 52-60. doi:10.1016/j.displa.2017.01.001

[24] Cardoso, A. A., \& Vieira, F. H. T. (2018). Adaptive estimation of Haar wavelet transform parameters applied to the fuzzy prediction of network traffic. Signal Processing, 151, 155-159. doi:10.1016/j.sigpro.2018.04.026

[25] Ch. HimaBindu, MaruturiHaribabu, K. VeeraSwamy Fusion Based Watermarking Scheme with Multi-Level DWT \&SVDInternational Journal of Engineering \& Technology, 7 (3.6) (2018) 270275 
Figures

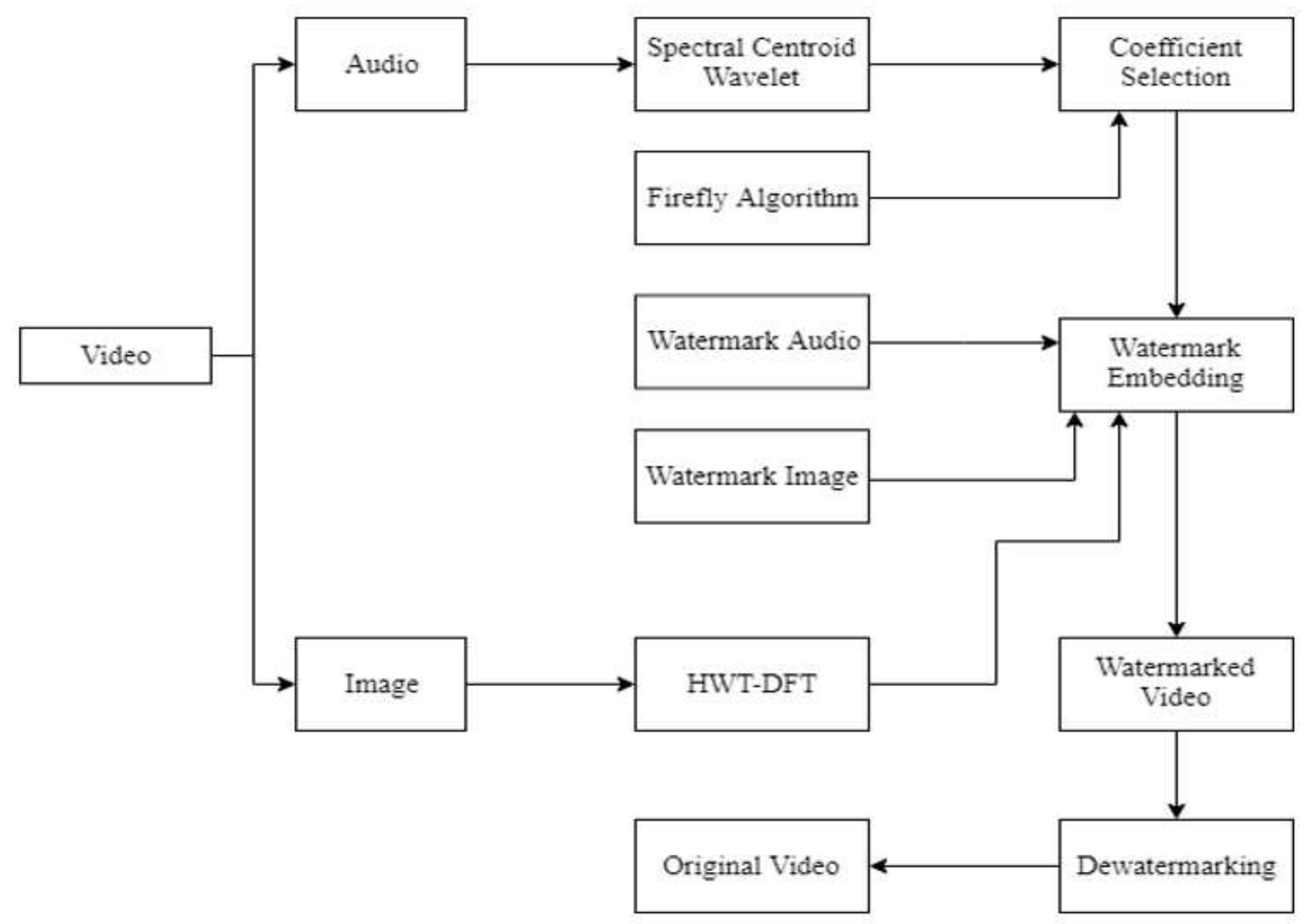

Figure 1

Watermarking Process 


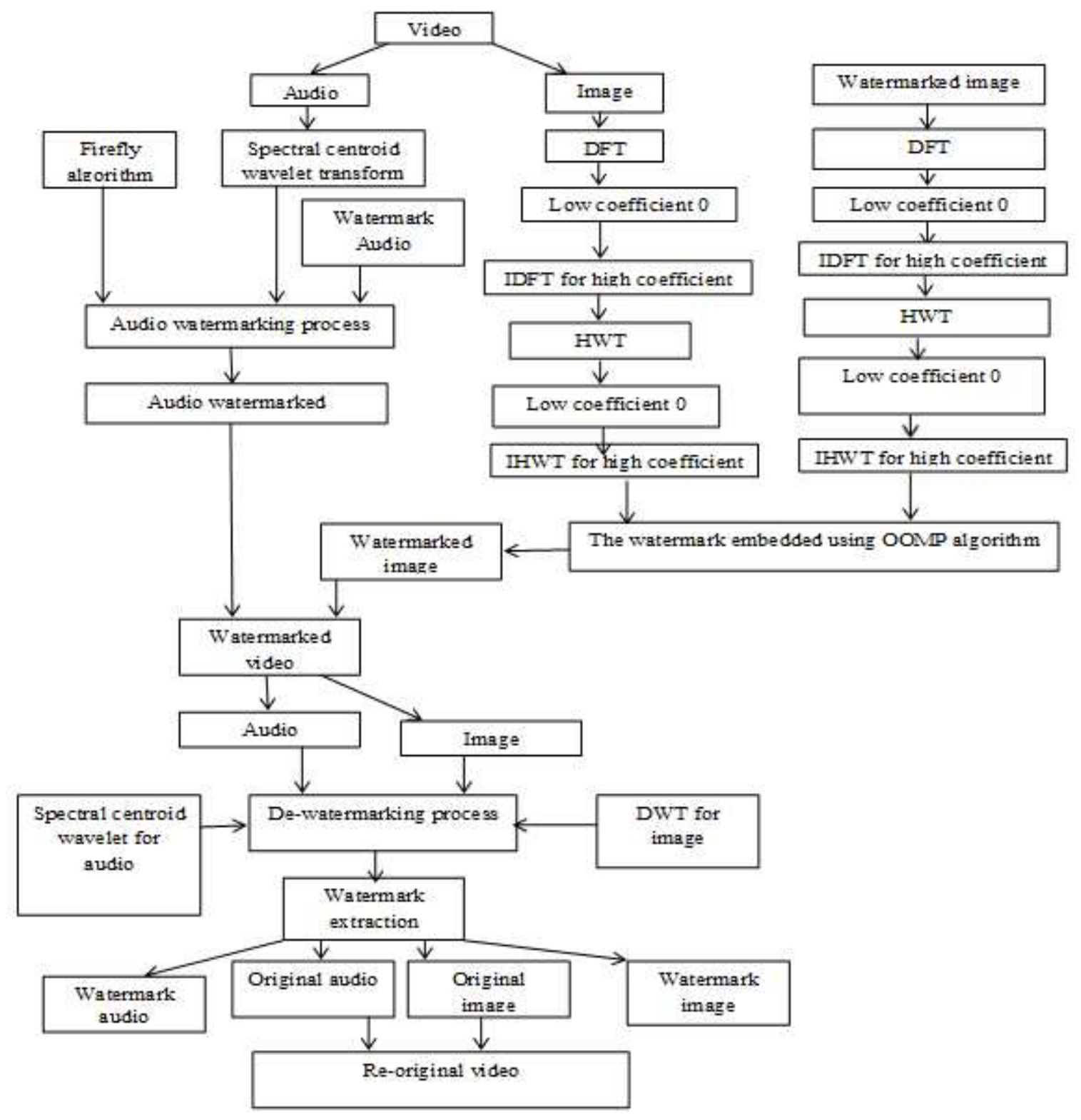

Figure 2

Flow diagram of Watermarking 


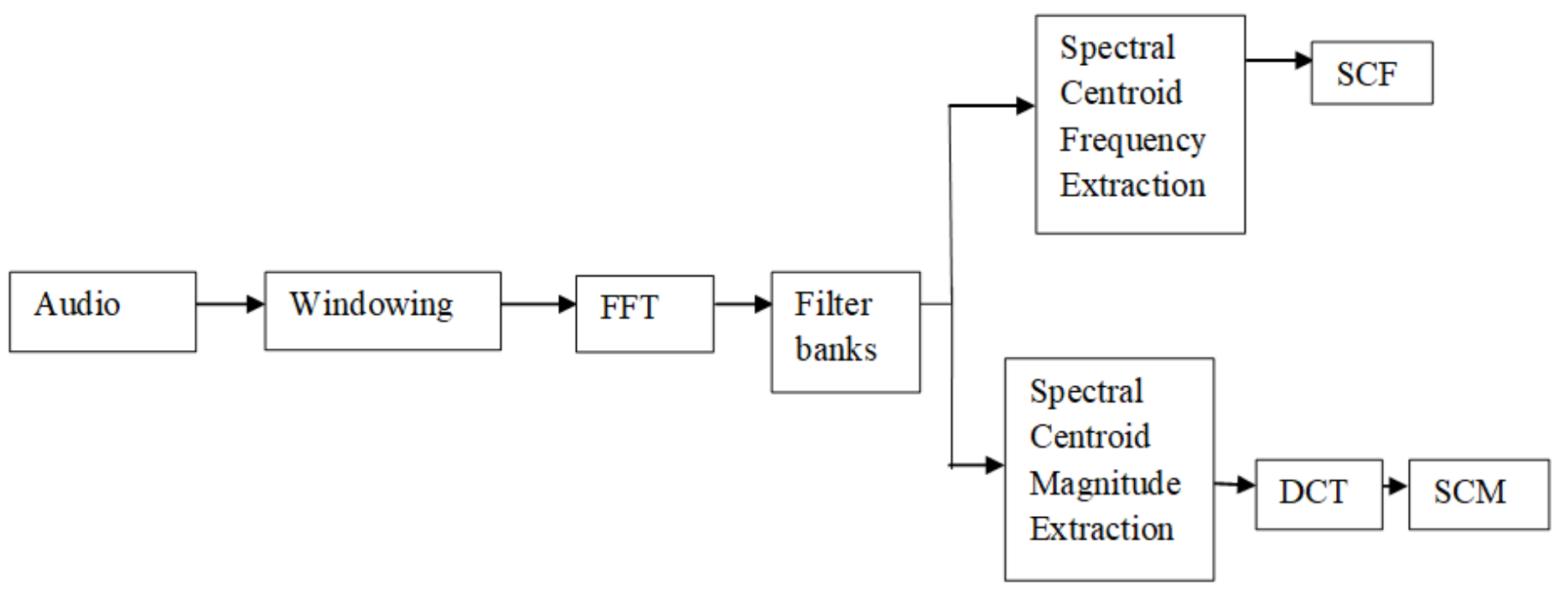

\section{Figure 3}

Block diagram of Spectral Centroid Frequency process

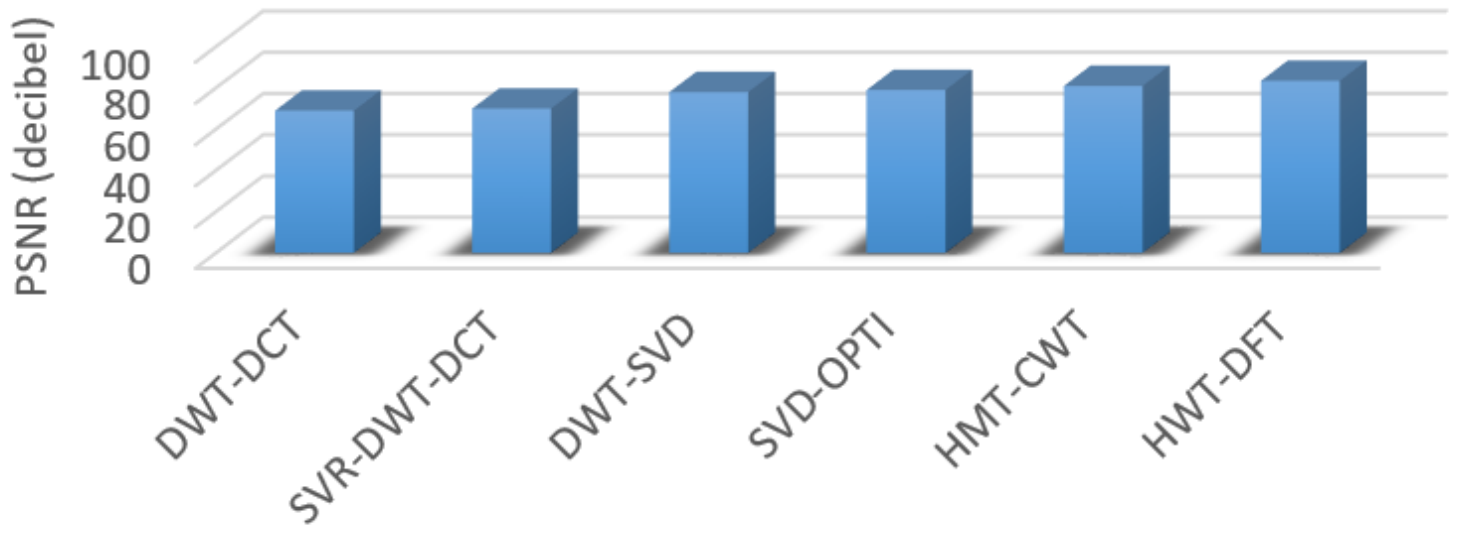

Different Watermarking Techniques

Figure 4

PSNR of Images

Attacks

Salt \& Pepper (0.0012)

PSNR

Gaussian $\mathrm{G}=0, \mathrm{~h}=0.000022$

52.341 
Figure 5

Performance of PSNR based on attacks

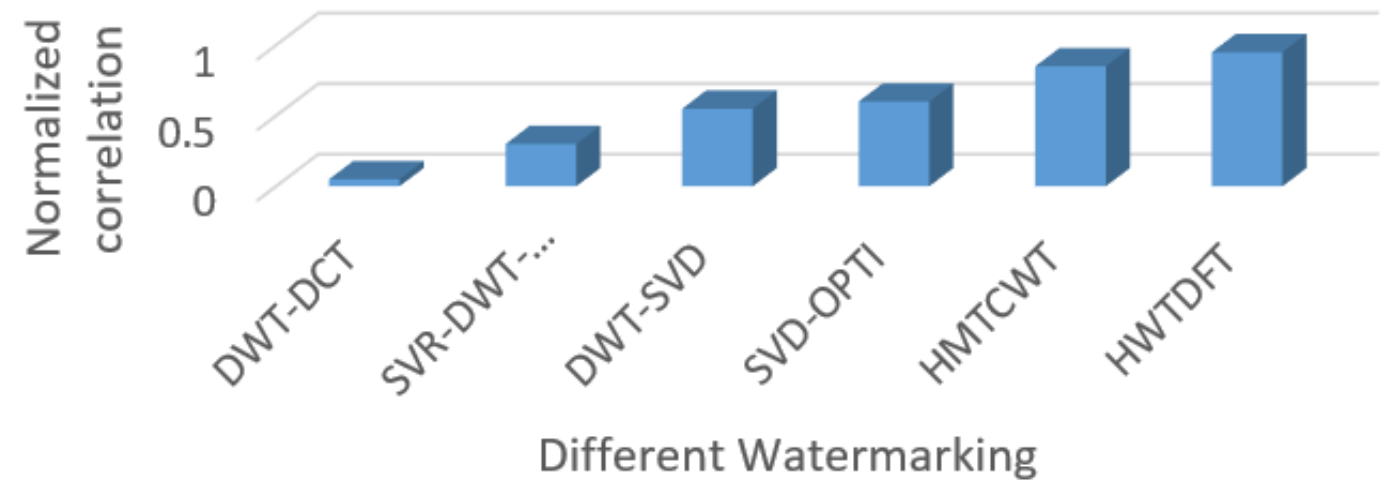

Figure 6

Normalized Correlation (NC)

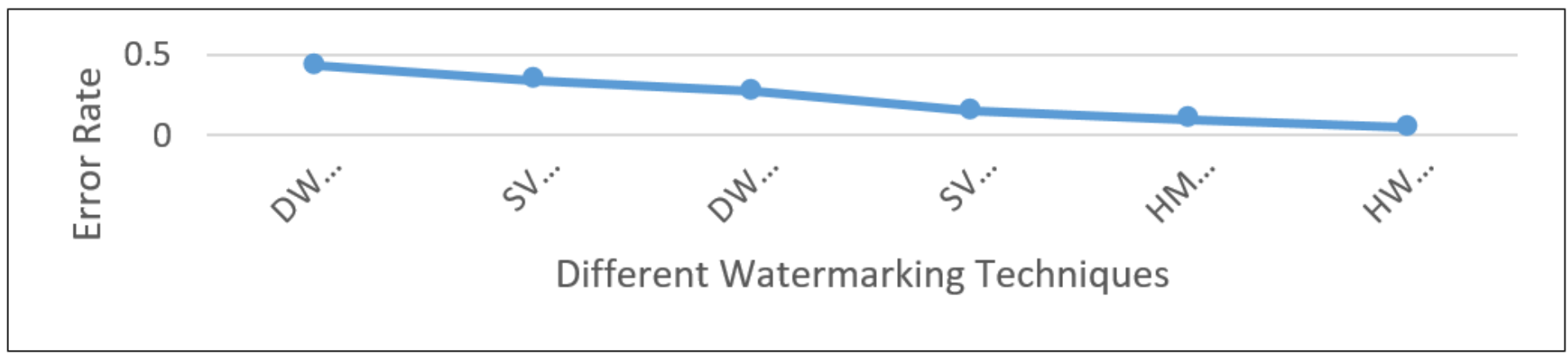

Figure 7

Mean Square Error

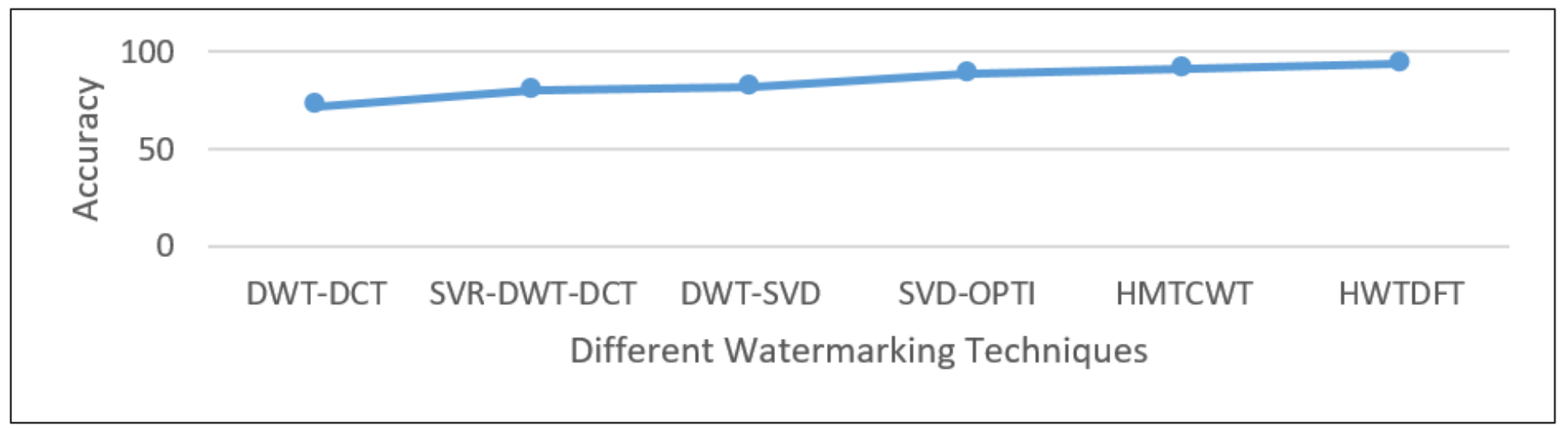

Figure 8

ACCURACY 
MSE

0.1

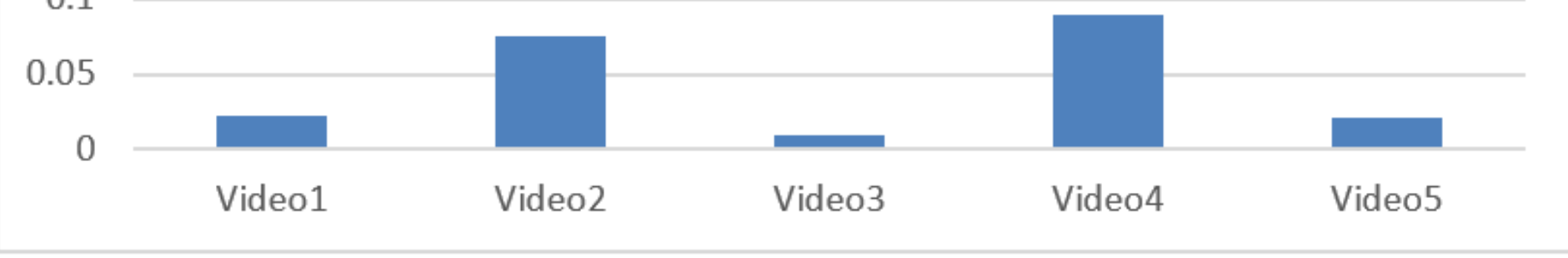

Figure 9

MSE of Images

\section{PSNR}

80

60

40

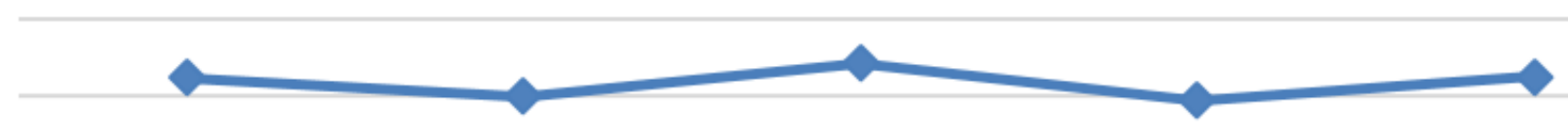

Video1

Video2

Video3

Video4

Video5

\section{Figure 10}

PSNR of Images

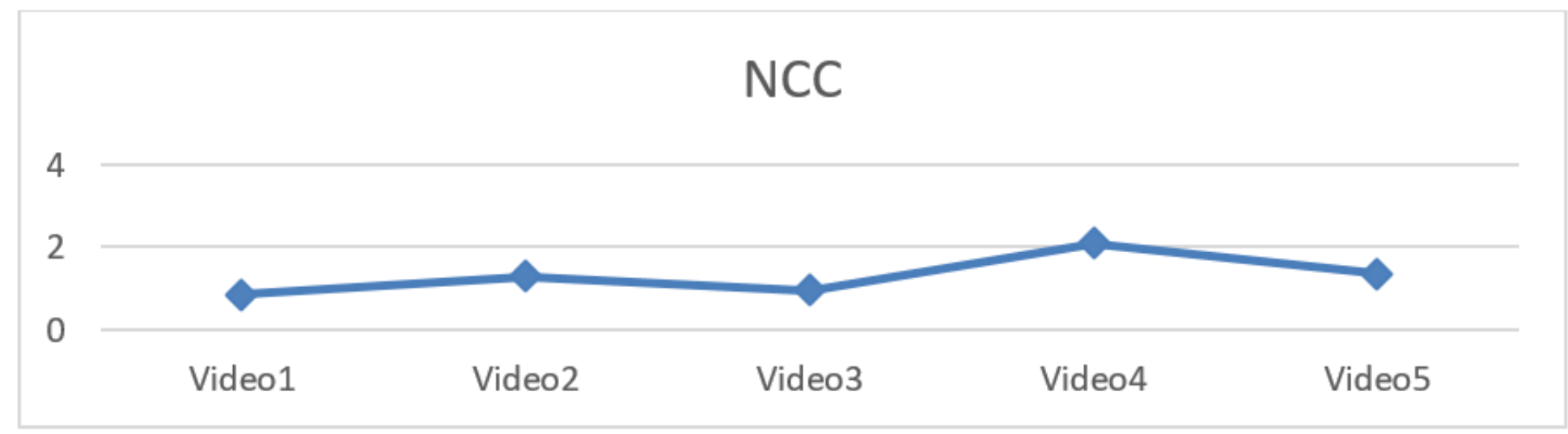

\section{Figure 11}

Normalized Coefficients of Images 


\section{SSIM}

1

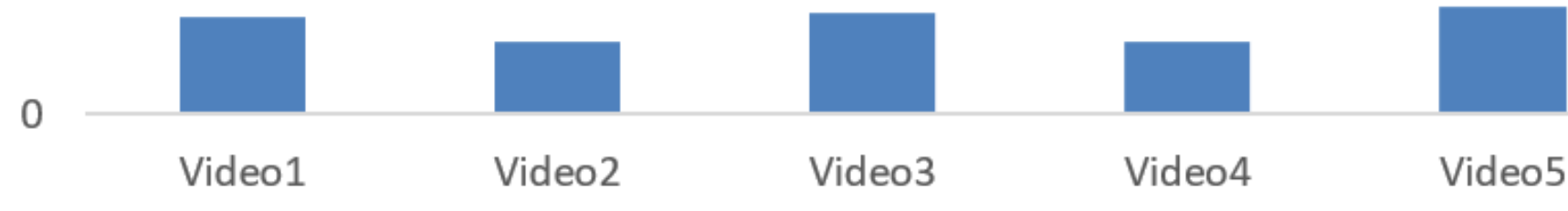

Figure 12

SSIM of Images

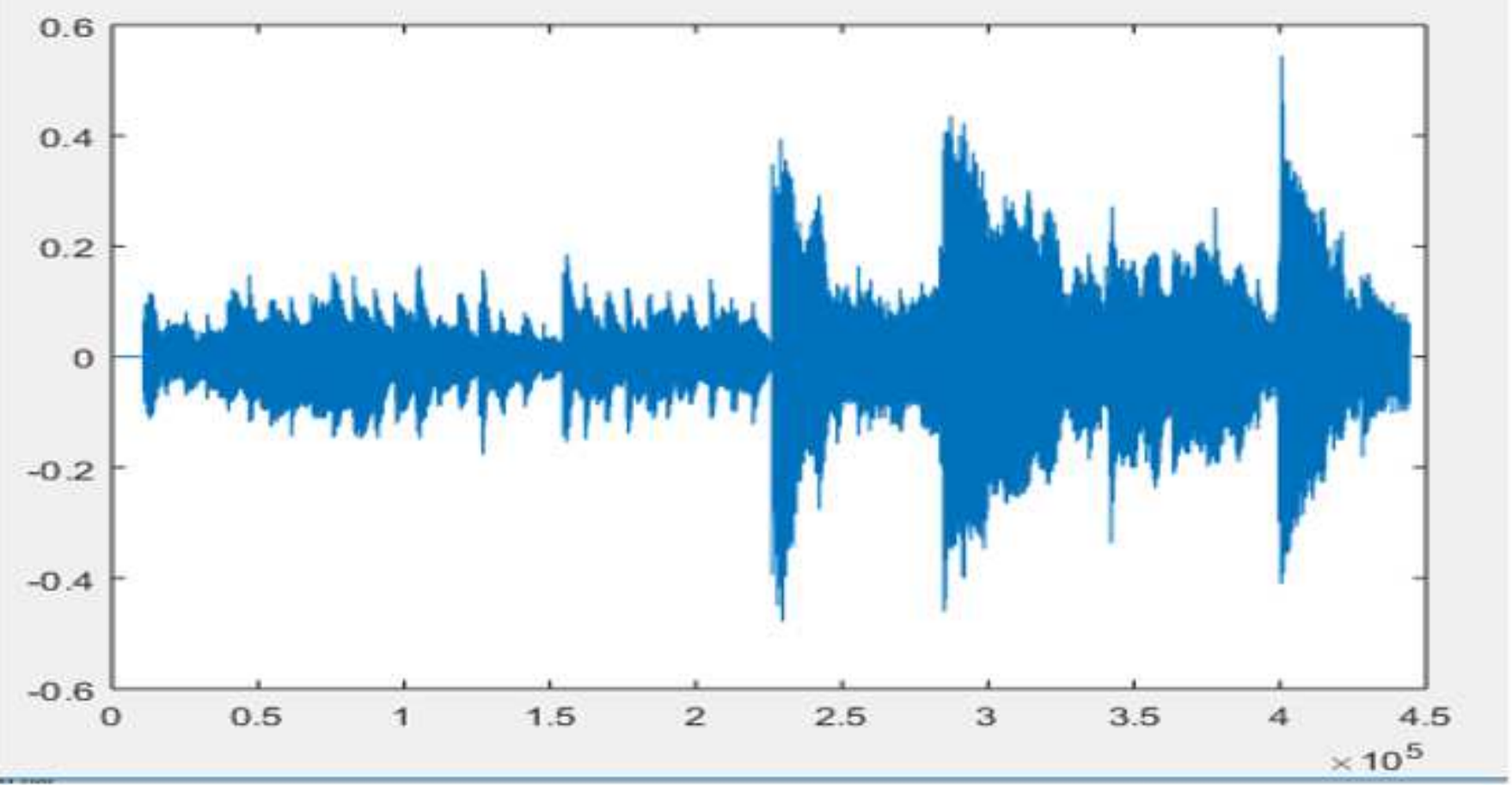

Figure 13

Input Audio signal 

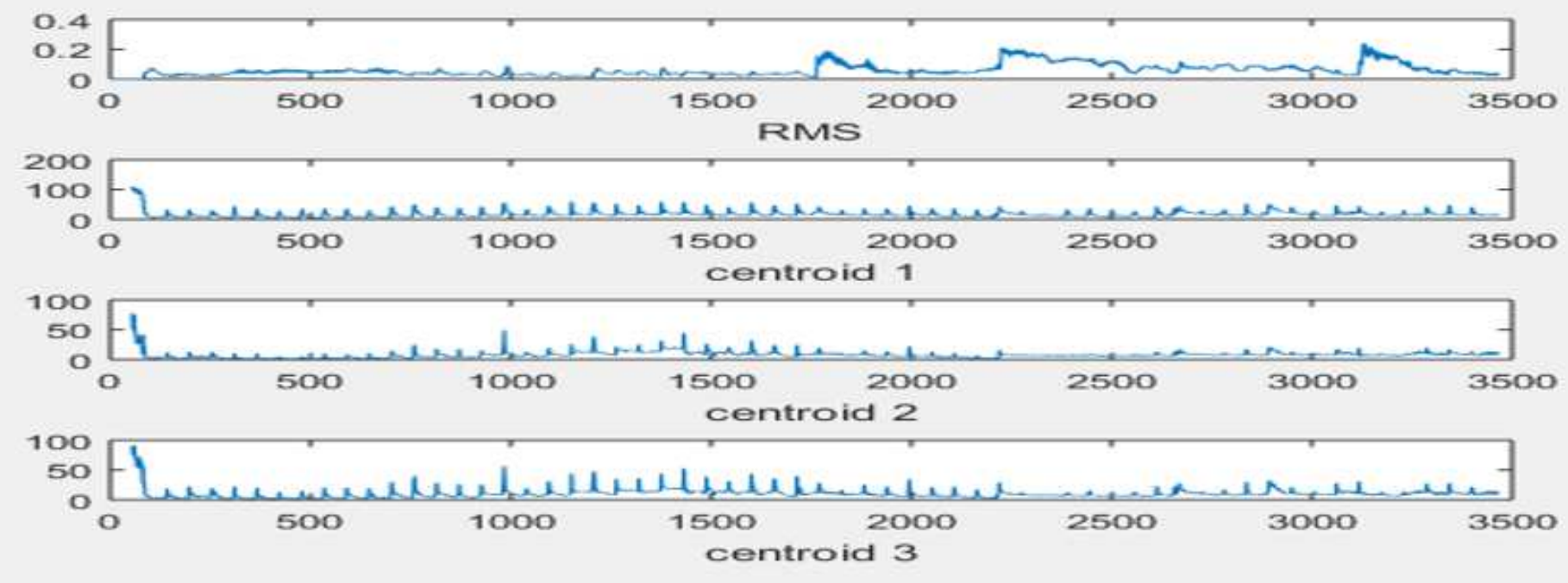

\section{Figure 14}

Graph of Audio signal using Spectral Centroid.

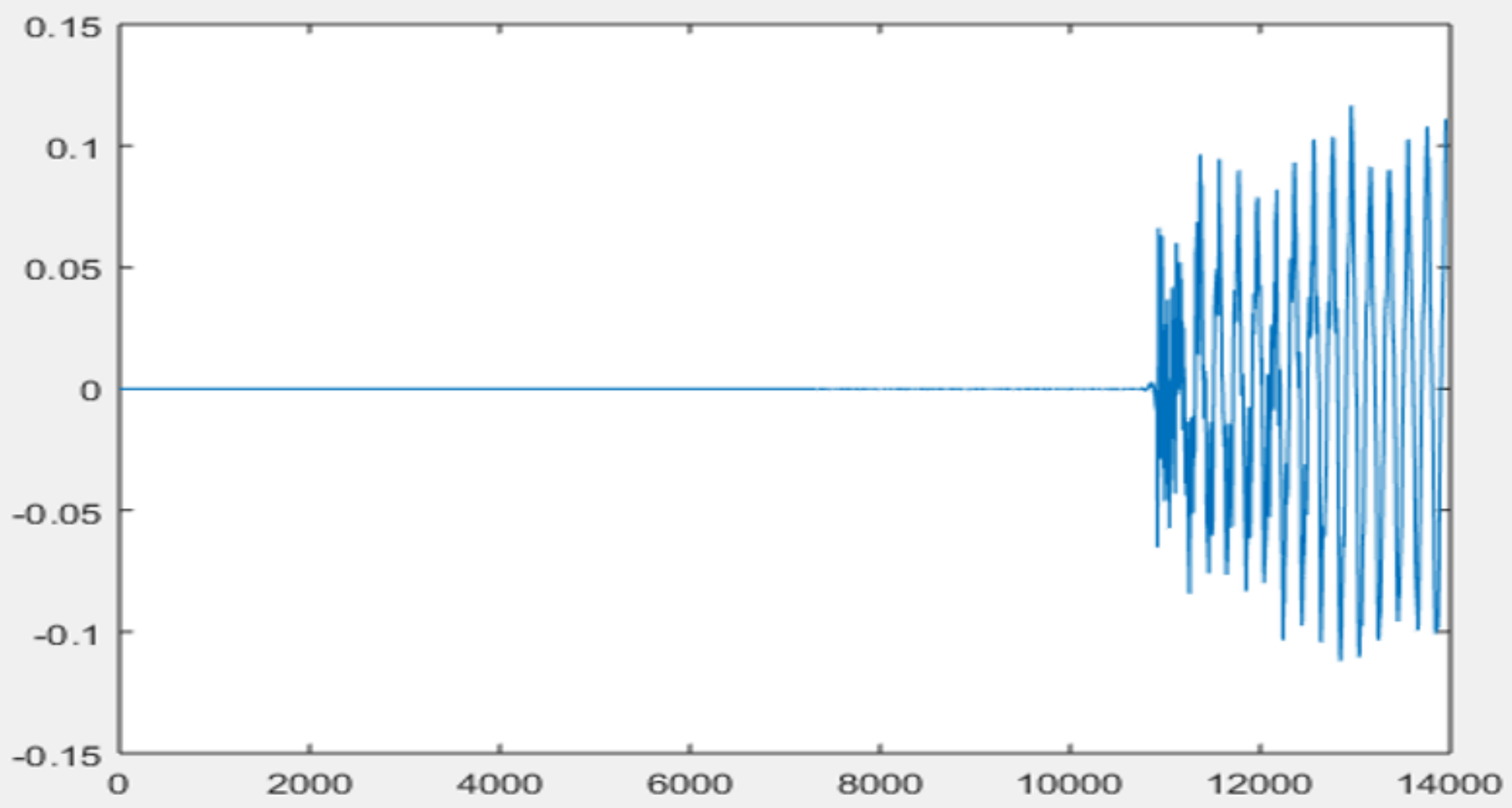

Figure 15

Shows the attack in the noise signal. Figure 15. 


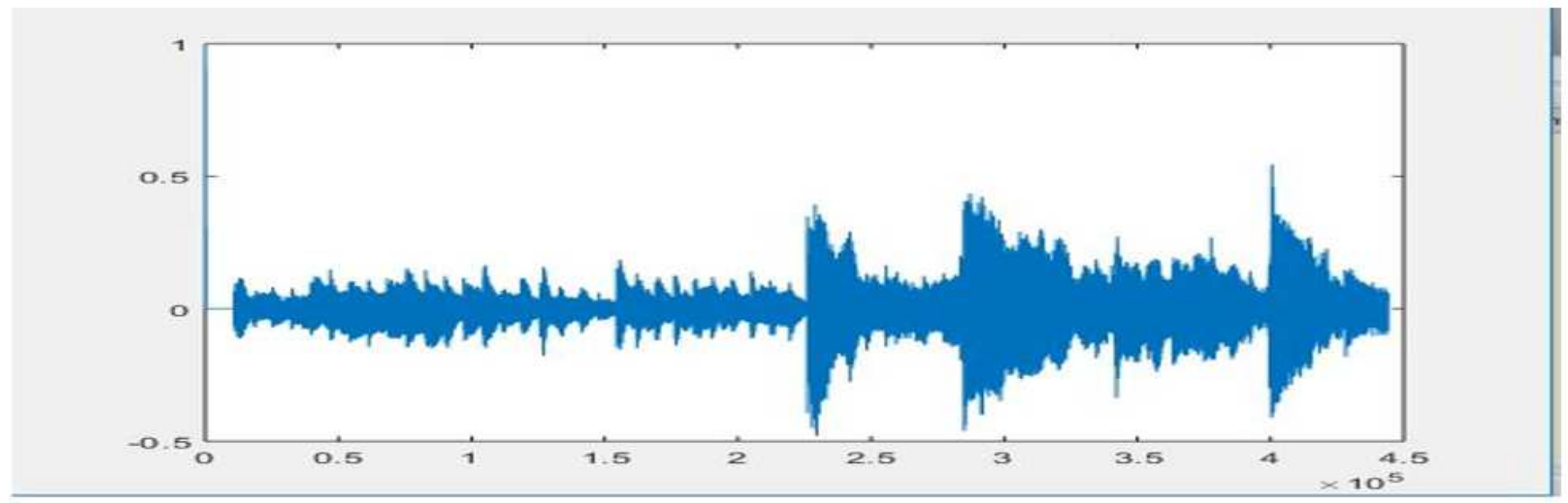

Figure 16

Watermarked audio 